\title{
Identified improvements of wireless sensor networks in smart grid: issues, requirements and challenges
}

\author{
Ines Hosni* and Noureddine Hamdi \\ National Engineering School of Tunis, \\ University of Tunis El Manar, \\ LR-99-ES21 Laboratory Systems Communications, \\ 1002, Tunis, Tunisia \\ Email: ines.hosni@hotmail.fr \\ Email: noureddine.hamdi@ept.rnu.tn \\ *Corresponding author
}

\begin{abstract}
Communications infrastructure is a basic part for the success of smart grid. Optimisation of energy consumption in the future intelligent energy networks (smart grids) will be based on the integrated near real-time between the different elements of grid network communications. Through a communication infrastructure, a smart grid can improve the reliability of power, eliminate power outages, and optimise energy consumption. The network gets smarter by improving detection, so the most important element that makes the smart grid is wireless sensor networks. Among them, one of the most important applications is a sensor data collection. This paper discusses some of communications research challenges and opportunities in the areas of smart grid and smart meters. In particular, we focus on some of the main communications challenges to achieve interoperability and future proof, smart grid/metering networks. We describe the basic taxonomy and propose to break the network data collection of wireless sensors.
\end{abstract}

Keywords: smart grid; communication infrastructure; wireless sensor network; WSN; communication technologies; smart metering; monitoring; sensing; sensor data collection.

Reference to this paper should be made as follows: Hosni, I. and Hamdi, N. (2016) 'Identified improvements of wireless sensor networks in smart grid: issues, requirements and challenges', Int. J. Smart Grid and Green Communications, Vol. 1, No. 1, pp.3-37.

Biographical notes: Ines Hosni received her Engineering diploma in 2012 from the Institute of Applied Sciences and Technologies (INSAT) of Tunisia, and her Master's degree in 2012 from Tunisia Polytechnic School (EPT). At present, she is working on her $\mathrm{PhD}$ on Communications System in the National School of Engineers (ENIT), Communications System Departement, LR-99-ES21, Tunisia.

Noureddine Hamdi received his Electrical Engineering diploma in 1993 and his Master's degree of Science (DEA) in 1994, and his Doctorate thesis in Electrical Engineering in 1999, all from the National School of Engineers of Tunis (ENIT). From 2000 to 2012, he was an Assistant Professor until 2007, then an Associate Professor, in the Institute of Applied sciences and Technologies (INSAT) and Tunisia Polytechnic School (EPT). Currently, he is a Professor in the Institute of Applied sciences and Technologies (INSAT), and the Director of Engineering Studies in Tunisia. 


\section{Introduction}

Smart grid is a term referring to the next generation power grid in which the distribution and management of electricity is improved by incorporating a two-way communications and capabilities pervasive computing for better control, efficiency, reliability and security. A smart grid delivers electricity from suppliers to consumers using two-way digital technology. It controls the intelligent homes or buildings devices, reduces costs and increases reliability, efficiency and transparency (US Department of Energy). It is expected to be a smart grid to modernise legacy grid. It provides monitoring, protection and automatically optimises the operation of interconnected elements. Thus, smart grid is an intellectual and logical electricity network that integrates the actions of all users connected to it and makes use of bidirectional electricity flow and information, sophisticated and highly advanced control, and communications technologies to rescue energy, reduce cost and increase reliability. In the smart grid, timely and reliable information becomes the key factor for reliable delivery of power from the generation units to the end users. It integrates the advantages of modern communications to provide real-time information and enable the near-instantaneous balance of supply and demand.

Smart grid has an upgrade of existing power networks with advanced information and communications technologies (ICTs), intelligent appliances and systems. Many ICT technologies that must be adopted by smart grid have already been used in other industrial applications, such as sensor networks in manufacturing and wireless telecommunication networks, and are designed to be used in the new smart and interconnected paradigm shift. Such technologies are enabling two-way communication, interaction between users and the electricity market, grid monitoring in real time, flexibility to changing situations, optimal use of resources and equipment, management and forecasting of electric power consumption, integration, monitoring, control, maintenance, security against attacks and threats, and etc..

Communication technology of smart grid can be grouped into five main areas:

- Developed devices: take an active role in determining the electrical performance of the grid. They may be applied either in autonomous or interconnected applications to build complex systems such as microarrays. These devices are based on basic research and development gains power electronics, superconductivity, materials, chemistry and microelectronics.

- Developed control methods: present devices and algorithms that will analyse, diagnose and prescribe the conditions in modern grid and identify and take appropriate remedial action to remove, mitigate and predict failures and disruptions to power quality.

- Sensor and measurement: is an essential component of a completely modern grid. It will purchase and transform data into information and improve several aspects of the power management system. These technologies will assess the health of the device and the integrity of the grid. They will support frequent meter readings, remove estimated billing, and prevent energy theft. They will also help relieve congestion and reduce emissions by allowing the choice of consumers and demand response and supporting new control policies. 
- Enhanced interfaces: critical technologies that must be implemented if the network operators and managers must have the tools and training they need to run a modern network. It will convert the data from complex supply network information that can be understood by human operators at a glance, animation, colour contours, virtual reality, and other technical data view, will prevent data overload and help operators to identify, analyse and act on emerging issues.

- Incorporated communications: a basic need that is required by other keys technologies for the modern power grid. This technology will create a dynamic and interactive mega infrastructure for real-time information and the sharing of power, allowing users to interact with several smart devices in an integrated system susceptible to various speed requirements (including near real-time) of the interconnected applications.

Figure 1 Smart grid communication infrastructures

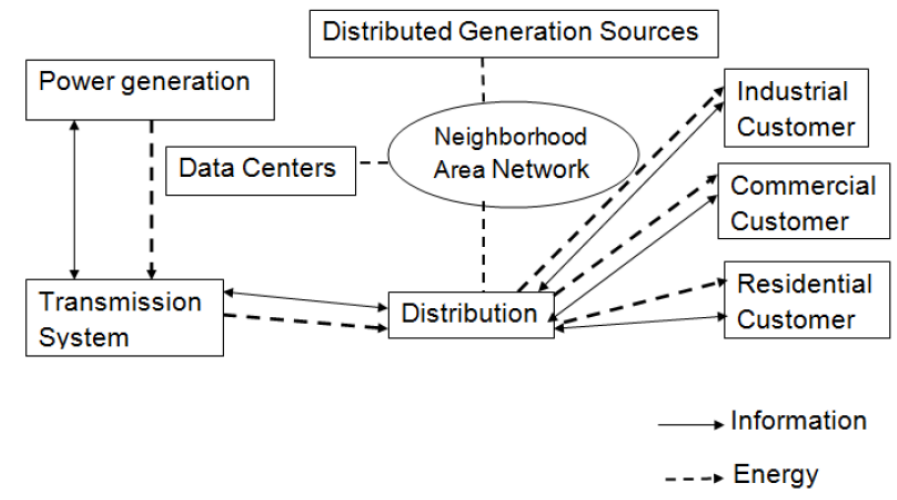

Figure 1 illustrates a general architecture for smart grid communication infrastructure, which includes the home area networks (HANs), the business area networks (BANs), the neighbourhood area networks (NANs), data centres, and station automation integration systems (Yu et al., 2011). The intelligent system infrastructure consists on energy, information and infrastructure underlying communication that supports smart grid (Fang et al., 2012; Lo and Ansari, 2012). Smart infrastructure can be divided into three subsystems: the smart energy subsystem responsible for advanced electricity supply and consumption production, such as power generation, transmission grid, distribution grid, New grid paradigm: microgrid and grid-to-vehicle/vehicle-to-grid (V2G). The smart information subsystem responsible for advanced information metering, monitoring and management measures such as: smart meter, sensor, phasor measurement unit (PMU), data modelling and information analysis, integration, and optimisation. And the smart communication subsystem responsible for the advanced communication technologies and information transmission among systems, devices, and applications such as: wireless mesh networks, cellular communication systems, cognitive radio, satellite communications, microwave or free-space optical communications, fibre-optic communications, power-line communications, etc. 
The smart grid power distribution between producers and end-users using the bi-directional information is used to control smart appliances at consumers, save energy consumption and therefore reduce costs while increasing the reliability and transparency of the system by supplying more visibility for all consumers in their use and energy costs and allowing all consumers to participate in energy market. For example, in the traditional power grid, the electricity is produced by the generation plant, and then moved by the transmission grid, the distribution grid, and finally delivered to users. In smart grid, electricity can also be go back into the grid by users. For example, users may be able to generate electricity by utilising energy solar panels at homes and put it back into the grid, or electric vehicles (EV) can provide energy to help balance loads by 'peak shaving' (sending power back to the grid when demand is high). This backward flow is important. For example, it may be extremely useful in microarray which was 'islanded' due to power failures.

Intelligent measurement techniques/capture can provide energy in real time, when the detected data are sampled continuously at some or all of sensor nodes, thanks to a smart meter which is a digital meter that is located at a home or business to measure the amount of electricity used. It reads and stores the electricity consumption over short intervals, typically every 15-30 minutes, and remotely sends this information to the network distributors and retailers daily. It has the potential to change people's relationship with energy, from a passive one in which people receive an invoice once a quarter, to an active one in which they can access almost real-time information on energy consumption so they can analyse and understand their use. A concentrator (gateway) collect data packets from smart meters using short-range communication technologies (WiFi or ZigBee). The received packets are stored in the buffer of the concentrator. After the data packets sent from concentrators are received by the base station which is in-charge of bandwidth allocation for the data transmission of each concentrator. Data are then forwarded over internet to the control centre for processing and storage.

The network operations centre may retrieve the customer power consumption data to maximise power production, and ensuring the distribution function of the energy consumption. In a complex system of smart grid, through the deployment of new components and the existence of information technology and control, it can provide sustainable activities for utilities and customers (Amin and Wollenberg, 2005). It can also improve the legacy efficiency of the generation distribution systems, transmission and penetration in the use of clean and renewable energy by introducing modern communication systems in smart grids. The new communication infrastructures must evolve for the transmission of data almost everywhere capable of handling delivery applications with large amount of new data from smart grid applications. These networks should be scalable to support the present and future of all functions characterising the platform of smart grid and smart technology communication network.

We discuss several key factors for smart grid systems, we then discuss issues and prior solutions on wireless sensor network (WSN) deployment and data delivery protocols.

The remainder of this paper is organised as follows: in Section 2, we discuss some key factors for smart grid systems including power line communications (PLC), distributed energy resources (DERs), and some communication techniques and issues. Section 3 presents smart metering communications. and Section 4 discusses the opportunities to apply WSN in smart grid. Issues and solutions on the data delivery protocol design are investigated in Section 5. Section 6 summarise major requirements 
that smart grid communications must meet and Section 7 explore the challenges for a smart grid. Finally, Section 8 concludes the paper and gives further discussions on the directions of future work.

\section{Communication techniques and issues}

\subsection{Power line communications}

Communications over power lines (PLS) is an old idea that since the early 1900s, when the first patents were made in this area (Schwartz, 2009). Since then, electricity utilities around the world have been using this technology for remote measurement and load control (Ferreira et al., 2010a), using the initially narrowband carriers solutions (NB) operating in the audio/low frequency bands that have achieved data rates ranging from a few bps to a few kilobits per second. As technology has advanced and the application space has expanded, the PLC broadband (BB) systems operating in the high frequency band $(2-30 \mathrm{MHz})$, increasing the data rates up to $200 \mathrm{Mb} / \mathrm{s}$ began to appear on the market. In recent years, the interest of industries has also increased around the broadband NB-PLC data based on multi-carrier systems operating in the band between 3-500 kHz. PLC is also used to provide internet access to residential customers, BB LAN connectivity within the home/office/vehicles, command control capabilities for automation and remote sensing. The basic incentive for the use of the controller is that the electrical network provides infrastructure which is much larger and pervasive any other alternative/or wireless, such that substantially each power line may become the target service value added. PLC is a technology for transporting a data driver, as used for the transmission of electric energy. In recent decades, utility companies was used for PLC for remote metering applications (Ferreira et al., 2010b). The discussion on what is the real role of PLC in the future smart grid is always open. Some argue that PLC is a very good candidate for some applications (Galli et al., 2010; Rieken and Walker, 2011), while others raise concerns about PLC (the issue of security due to the nature of high-voltage lines) (Pavlidou et al., 2003). Although smart grid can use different communications technologies, definitely, the PLC is single wire technology deployment cost compared with other wireless technologies (Galli et al., 2010). Even though the most effective way to use PLC is not yet clearly, Galli et al. (2010) predicted that the PLC may be more appropriate to the distribution network. Traditional stations in the middle voltage distribution networks are not equipped with communications capabilities. Thus, using existing power line infrastructure shows an attractive alternative to the establishment of new communication links. This allows information state and event flow between positions within a network. PLC uses the power line network as a means of communication. First generation systems ripple control provide one-way communication, in which centralises the load control and clipping have been made for many years. The third generation systems based on OFDM with rates much higher data are being developed and deployed for smart grids, for the distribution automation and advanced metering management. With the development of smart grids, the PLC transmission have become one of the possible technologies for information exchange between end users and utilities. To provide communication services with different priorities intelligent network environment, it is essential to design a PLC system with variable data rates supported. 
Test scores in Zhai (2011) indicate that the principal reason affecting the high speed communication on the supply line is the attenuation of the high frequency signal. There is practically impossible to use the frequency range of $10-20 \mathrm{MHz}$, it must be solved by means such as the repeater and modulation schemes. Except that the power cables are not designed for data, they are also prone to be interfered by the result of the inverter. Consequently PLC modems developed for household applications may not work. Most recent contributions have discussed PLC for high data rate applications such as internet access or multimedia communication. However, it lacks the consideration with PLC as a catalyst for the detection, control and automation in large systems comprising tens or hundreds of components spread over relatively large areas.

\subsection{Distributed energy resources}

It is generally believed that the growth of energy demand has exceeded the speed at which the energy can grow by traditional means. In addition, many governments agree that gas emissions must be contained to control or prevent climate change. The need of modernising the power grid infrastructure around world is both a consequence of the limited investment made in recent decades, and the result of new needs arising in the safe integration of public services renewable scale feeding transmission system, DERs feeding into the distribution system or the home. In fact, The legacy of power generation and transmission concept are converts to massive distributed energy incorporating a large number of variables and small renewable energy resources (DER), as the wind production (Wiser and Bolinger, 2008) - solar (Albany, 2005) facilities with all their stimulating effects the smart grid. MetaPV (Van Loon et al., 2010) is a project that has demonstrated the benefits of supply electric photovoltaic (PV) on a large scale, pointing the way to the city powered by renewable energy sources. The project also shown an implemented control capabilities in PV inverters, including the control of active power, the exploitation of autonomous network, and the distribution interaction monitoring system with PV systems. Intelligent control should allow an increase in PV penetration into existing power grids and encourage the use of renewable energy sources in cities and industries at minimum additional investment costs. Innovative monitoring and control concepts are needed to run these DERs safely and reliably, so that communication technologies must support it. A key requirement to facilitate the production of future distributed grids is that communication and information are standardised to ensure interoperability. For example, IEC 61850 , which was initially intended to automate substation, has been extended to cover the monitoring of DER. An important observation made in Infield and Li (2008) is that the existing electrical networks were designed in one direction radial mode regardless of the communication with the emergence of distributed generation from renewable resources. Sauter and Watson (2007) discussed the wider implications of the social acceptance of this new generation of energy technologies, because they represent a major departure from the traditional historical approach to the production of monolithic large-scale energy. In addition, the impact of regulatory and economic aspects also contribute to the recovery potential and different deployment templates to increase the adoption of these generators of renewable resources allocated. Each DER includes an electronic power processor (EPP) to regulate the exchange of energy with the smart grid and switching power interface (SPI) to monitor the currents rely from smart grid. Such PPE distributed and SPI should do together to take full advantage of smart grids power. To reach this goal, different approaches can be used, 
under the provision communication capacity. Tenti et al. (2010) considered different solutions to specific default enslavement of a supervisory control, for example, in residential micro-networks, where communication is possible between neighbours units or not available at all.

\subsection{Self-organisation overlay networks}

Due to the magnitude and complexity of deploying smart grid, telecommunication networks systems could lean on what already exist in public networks, such as wireless and fixed access technologies and the private networks. The goal of these networks can be considered not only as a means of communication to exchange information and monitor control, but also as a catalyst for new services and applications. In many respects, the complexity and heterogeneity characteristics of smart grid will be similar to that of a radio access network supporting wireless voice and data (Fan et al., 2013). However, the quality of service (QoS) requirements and load model will be markedly different from those of a voice/data mobile typical network. Both will split, at least partially, the problems linked to the management and operation of a complex and heterogeneous network in which tasks such as network planning, operation, management functions, and network optimisation are significant. We think that the covered with a self-organising network on existing infrastructure could be the way ahead to support the wider deployment of smart grid systems. Such a network of self-organisation should assist features like resource discovery communication, negotiation and cooperation between the network nodes, the connection establishment and maintenance to provide performance guarantees required by the smart grid applications. Recently, new network architectures such as the cloud-based methods (Rea et al., 2013) have been put forward for the collection and control of smart grid data.

\subsection{Home network challenges}

Research into domestic networks has thus far covered supplying multimedia applications with high QoS, and transparent connectivity for home users. With the arrival of smart grid, new features and principles of system design must be considered. For example, envisage how to include smart meter or M2M gateway function into the home gateway in a profitable way (Sahu and Dehalwar, 2012). Obviously, the smart meter adds a new dimension to home networks, which complicates the matter of interference management and resource provisioning. With potentially every device in the home that provides information related to energy smart meter, it is easy to provide a command to increase the size of the number of devices in each home that can communicate with each other and with the outside world. Today homes can have two to three computers (desktop, laptop, smart phone) connected to the home network and to the internet. The tomorrow smart grid could have 20-30 or more appliances and devices connected to the same network. While the preferred (wireless) network for these appliances have not yet been established, it is clear that there will be far more devices connected depending on the network used. Although there have been many community discussion over the years to have "an IP address for every possible peripheral" at home, the convergence of energy supply and communication can be the catalytic converter that actually becomes a reality. In addition to many new devices that will be connected to home networks, new types of applications will likely emerge. The first application is the monitoring of energy consumption at home 
and other sectors (offices, etc..). In this sense, there are proposals for load monitoring and real time control of perspectives utilities. However, the power monitoring has the potential to develop into something much more important that the measurement of the energy consumed. With common worries about climate change and the need for very large energy efficiency in all fields it follows that the granularity followed by energy consumption at home and other areas becomes a necessity and much research will be needed to automation methods for reducing energy consumption in the house. Since there are a lot of waste collected in the manner appliances and devices that are used today (e.g., leaving appliances on standby, the inefficient use of washing machines and refrigerators, inefficient use of heating and cooling), there is many possibilities for the realisation of automated methods to reduce energy consumption.

\section{Smart metering communications}

The evolution of smart grid depends not only on advancing technology power equipment, but also the improvement of sophisticated computer monitoring, analysis, optimisation, and control of central utilities exclusively places for distribution and transmission. Many of distributed automation concerns should be addressed from a standpoint of information technology, such as data interoperability, exchange and integration with existing peripherals and future systems and applications. Therefore, an intelligent information subsystem is used to support the production of information, modelling, integration, analysis, and optimisation in the context of the smart grid. In this section, we focus on intelligent information. We first explore the extent of the information then we explore the monitoring unit and smart metering, generating information from end entities (e.g., smart meters, sensors). This information is often used for invoicing, network status monitoring and control of the user device.

\subsection{Smart metering}

Smart metering is the largest mechanism used in the smart grid for getting information by terminating devices and user devices, while checking the conduct of the features. Automatic metering infrastructure (AMI) systems (Hart, 2008) which are in turn constructed on automatic meter reading (AMR) systems (Rieken and Walker, 2011), largely considered as a logical strategy to reach smart grid. AMR is the technology of automatic data collection, use, and status data from energy metering devices. It transfers data to a central database for invoicing, troubleshooting and analysis. AMI varies from traditional AMR in enabling bidirectional communications with the meter. That is why almost all information is available in real time and on demand, improving the functioning of the system, the power of customer and managing demand (Fang et al., 2012).

Smart meter, is an advanced meter which identifies power consumption in much more detail than a conventional. It supports bi-directional communication between the meter and the central system, and sometimes considered as part of the AMI.

A smart meter is an electric meter which generally consumes information at intervals within an hour and sends this information at least daily public service for monitoring and invoicing purposes (Federal Energy Regulatory Commission, 2010). In addition, a smart meter has the ability to disconnect and reconnect away control devices and using devices to manage workloads and requirements in the future 'intelligent-building'. 
Figure 2 shows a typical usage scenario smart meters.

Figure 2 An example of smart metering structure

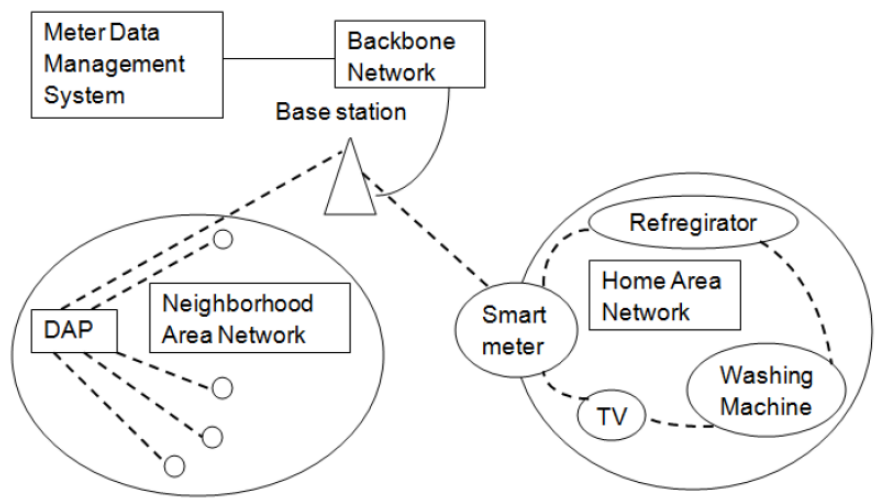

Other components of smart metering communication are HAN which is a network of information and communication consisting of equipment and devices in a home to support different circulated applications, NAN that gathers data from several HANs and outputting the data to a data concentrator, wide area network (WAN) is the transmission of data that carries the measurement data to the control centres, and gateway which is the device that collects and measures the energy consumption of the members of the HAN and transmits these data to concerned sides.

From the consumer point of view, smart metering provides a number of potential earnings. For example, end users are able to estimate and manage their invoices and reduce energy consumption invoices. From a public service perspective, they can use smart meters to achieve real-time pricing, which tries to encourage users to cut their application requirements, or to optimise the flow according the information sent by the demand side.

\subsection{Smart monitoring and measurement}

A significant according to the smart grid is monitoring and measuring of the grid conditions. Several systems have been set up to monitor and control power systems for decades. We quote the SCADA system (Jing et al., 2012). Experience within industries indicates that the convenience deployment of SCADA systems may limit the high voltage transmission only. Taylor et al. (2006) have made the observation that the monitoring and control existing systems are limited to the transmission network and inadequate to expand monitoring and control across the entire grid. A distributed control system monitoring is proposed to deal the power grid. A solution of grid computing is proposed to meet these purposes surveillance monitoring. SCADA systems develop; there is great interest in the investigation of security flaws posed to such systems on the network communication technology and internet. In Serizawa et al. (2010), the solution is to apply the system ICT in a hierarchical breakdown of the power grid into logical areas for surveillance and supervision. It describes the impact of the control centre responsible for the management and control of electricity network. It also provides a frame for future control centres to monitor and operate the smart grid. EU ADINE FP6 project (Samuelsson et al., 2010) is 
relied on the active network concept management (ANCM), where automation, ICT and power electronics are utilised for embedding more delivered generators by leveraging active resources rather than simply enhance the network.

In smart grid, there are two communication technologies: wired and wireless. Table 1 compares the different communication technologies. We discussed in the previous section the PLC which is a wired technology. It is also believed that these wired communication technologies will be included in SG. In addition to the PLC, we find the optical fibre which due to its high bandwidth capacity and immunity features. We believe that they will play a major role in the core networks of the information in the future SG (Gungor and Lambert, 2006). While it is well known that the cost of the slice of optical fibre can be expensive, but it is a profitable communications infrastructure for the castrate backbone communication networks in the future as these smart grid fibres are previously spread wide in today's communication network backbones, with a great amount reserve capacity used. Wireless technologies not only offer significant advantages over wired technologies, such as low cost of installation, rapid deployment, mobility, etc., but are also more suitable for remote applications. Wireless communication has already been widely used in our daily life and can be deployed anywhere and any time. Among the technologies of wireless communication can be applicable in the future SG, we will be interested in the WSN. Sensors or sensor networks have been used as monitoring and measurement approach for different purposes. For sensing mechanical breakdowns in power networks such as the driver failure, the towers collapse, hot spots, and extreme mechanical conditions, Leon et al. (2007) proposed that sensor networks should be incorporated into the power grid and help to evaluate the mechanical and electrical properties in real time, the conditions of transmission lines to obtain a complete picture of the physics and electrical power system in real time, diagnose imminent and permanent faults, and determine measures suitable control that could be taken automatically and/or proposed network managers once the extreme mechanical condition appears in a transmission line. In particular, the WSNs, given their low cost, can provide a workable and profitable detection and communication platform for remote monitoring system and diagnostic. Gungor et al. (2010) examined the application of sensor networks for power systems and their potential and challenges and presented a set of experimental studies in different environments power system. They concluded that, with the help of WSN, a one contingent system in the grid can be sensed and isolated before it causes a ripple effect and leads to more system failures. Other research on sensor networks for the purpose of ILI (Bressan et al., 2010; Lu et al., 2005, 2007). Bressan et al. (2010) explored the implementation of a monitoring system on a chip WSN, with particular emphasis on the establishment of a strong routing infrastructure using the routing protocol for low-power and lossy networks whose definition is currently under discussion within the IETF ROLL working group. Lu et al. (2005) has proposed a system of closed loop power management with a WSN, which was applied as an industrial system architecture of the plant energy management. The importance of the proposed system resides in its intelligent non-intrusive nature, and low cost. Later, Lu et al. (2007) extend previous work (Lu et al., 2005), and studied the whole system architecture. 
Table 1 Comparison of communication technologies for the smart grid

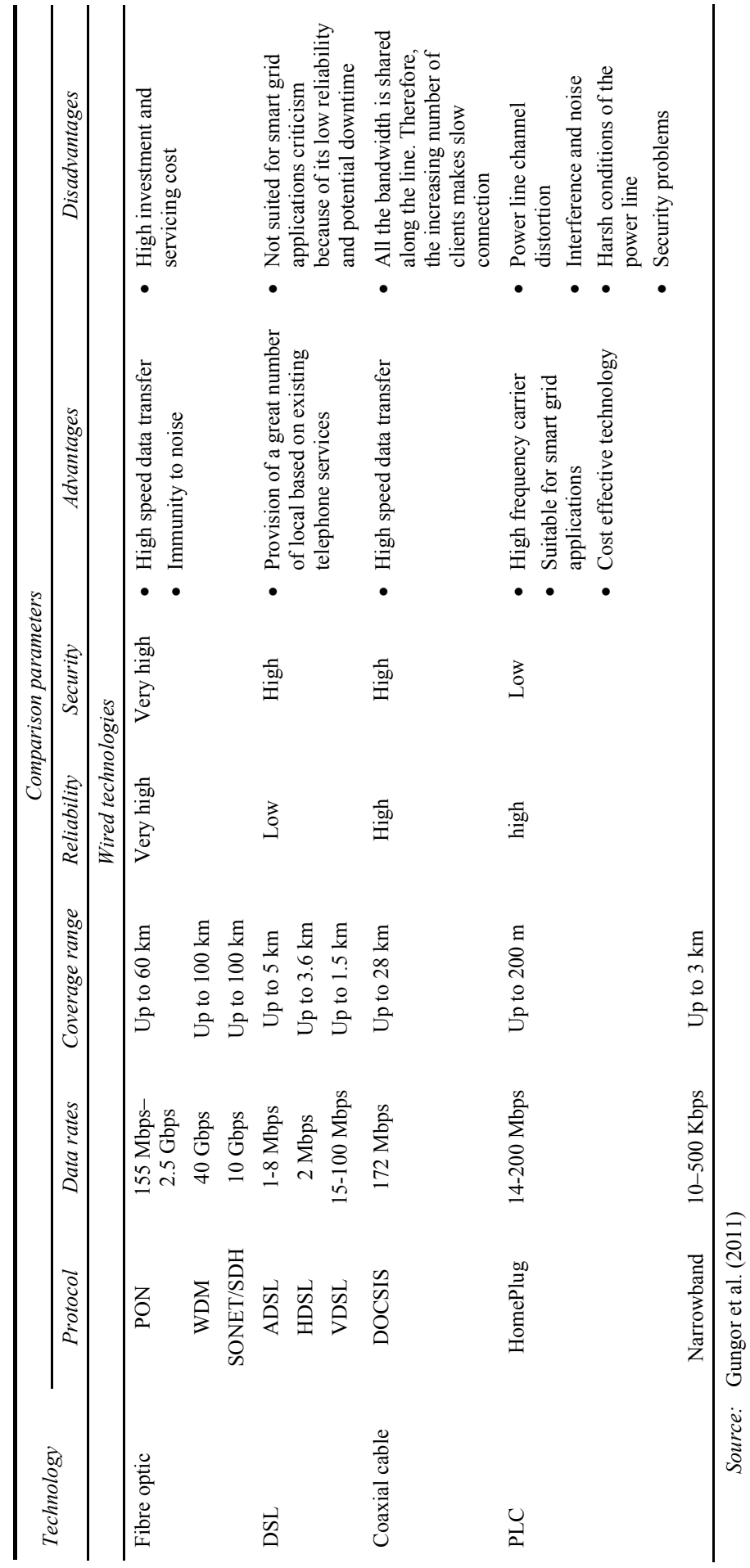


Table 1 Comparison of communication technologies for the smart grid (continued)

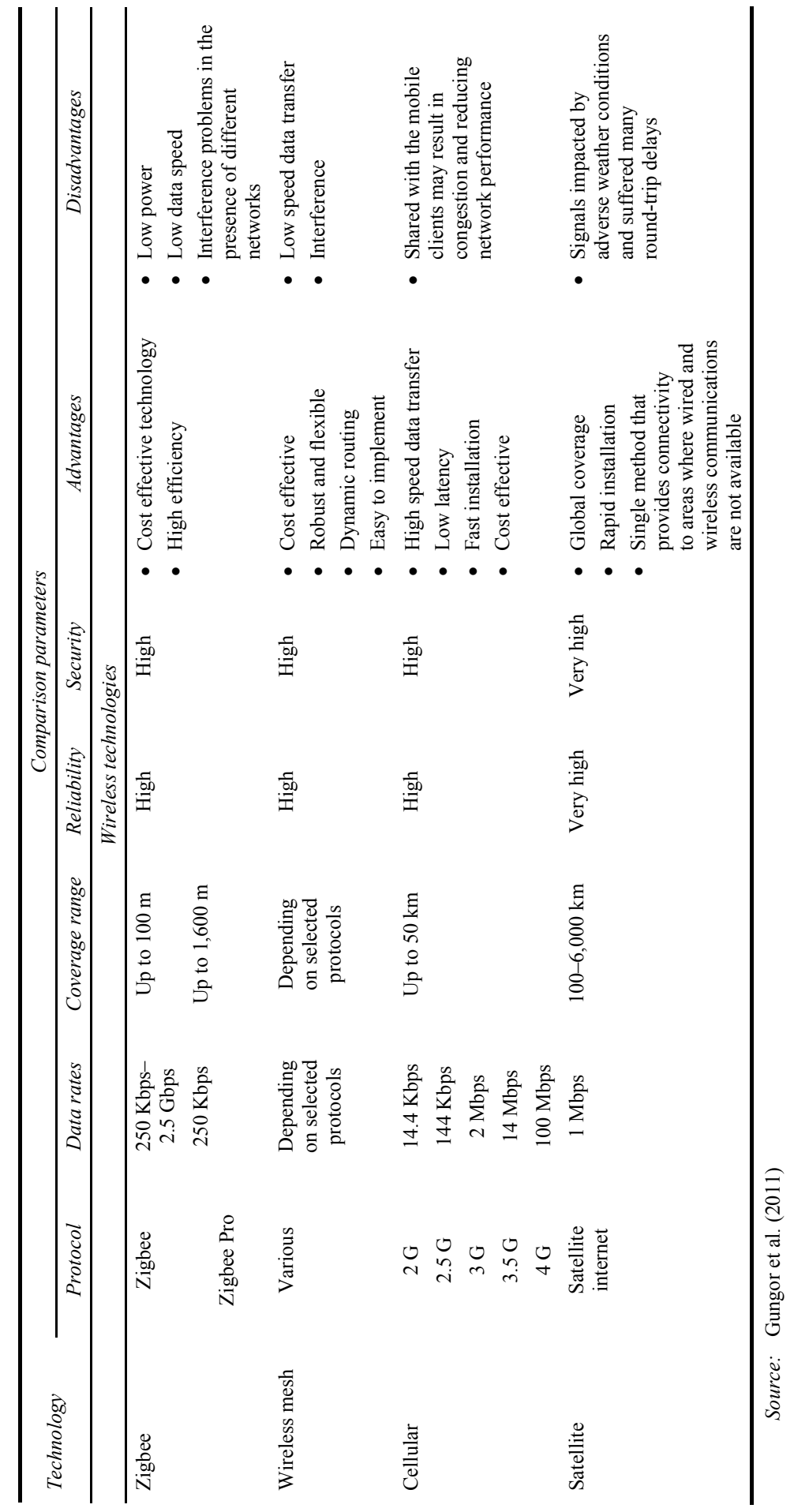




\section{Opportunities to apply WSN in smart grid}

In smart grid, reliable and online information becomes the key factor for reliable delivery of energy from generating units to end users. The effect of equipment breakdown, limited capacity, accidents and natural disasters, which cause electrical disturbances and failures can be avoided largely the on line supervision of the system supply state, diagnostics and protection. In this regard, the intelligent monitoring and low cost and control technology enabled line detection have become vital to maintain the safety, reliability, efficiency and availability of smart grid (Gungor and Lambert, 2006; Gungor et al., 2009; Lu and Gungor, 2009). The systems are generally made by wire communications. However, wired surveillance systems require cost communication, the cables must be installed and maintained regularly and thus, they are not widely implemented today because of their high cost. Therefore, it is necessary to have diagnostic systems that improve wireless monitoring and ensuring system reliability and efficiency by optimising the management electrical systems (Gungor and Lambert, 2006; Gungor et al., 2009).

Electrical networks contain three main subsystems: power generation, the supply of power, and power usage.

Recently, WSNs were largely renowned as a promising technologies that can improve these three sub-systems (Zhang et al., 2012), make WSN an essential component of the power generation system.

\subsection{Wireless sensor network}

As a newly emerging type of network, WSN has many peculiarities compared with conventional networks such as the internet, wireless mesh and ad hoc networks. First, a sensor node after it was deployed called to work for days, weeks, even years, free other interventions. As it is funded by the battery, efficient use of energy is required, which is dissimilar in internet networks, wireless mesh networks and mobile ad hoc networks, where sources of constant energy are available or the life expectancy is less than it is for WSNs. Although it is expected that a sensor node should work by a long period, it is frequently not necessary to work all the time, that is, it detects the surrounding environment, proceeds and transmits the data, then it idles for some time till the next cycle. In order to sustain fault tolerant, site is frequently wrapped by many sensor nodes. To prevent duplicate detection, while a node executes detection processing-transmitting cycle, other nodes are held in the vacuum state. In these cases, the power consumption can be reduced by leaving the inactive nodes turning to the dormant state, where most of the components (for example, the wireless radio, the detection component and the processing unit) in a sensor node are disabled. once the next round comes, these components are then woke up to return to the active state.

Duty ratio defined the ratio between the active and the period of complete rest. A small duty-ratio WSN are clearly has a lifetime much longer for operation. This function has been used in quite number of research (Gu et al., 2007). Another feature looked to the consumption of energy is to check the transmission range of a sensor node. Previous research has shown that one of the leading energy costs a sensor node comes from the wireless communication, where the major cost increases with the two to six capacity of transmission distance (Olariu and Stojmenovic, 2006). Consequently, the transmission range of a sensor node is frequently favourite to be adjusted and can be dynamically controlled to reach improved performance and low power consumption. 


\subsection{Sensor data collection}

In an application of sensor data collection, the sensors are usually deployed in areas characterised by the application requirement. Remote sensing data collected is then transmitted to a central base station for additional processing. Historically, such sensors are connected via wires which are used for the transmission of data and power. However, the wired procedure is the need for great lengths to deploy and maintain. To prevent disturbing other room temperature, implementation of wires should be carefully personalised, a failure in any wire may bring all off network and a lot of time and effort can be taken to find and replace the line string (Schelen et al., 2013). In addition, the tracking environment itself may render the wired deployment and holding very hard, if not impossible. For example, a local area of a volcano (WernerAllen et al., 2006) or wildfire stage (Hartung et al., 2006), where the hot gases and vapours may easily harm a thread. Even in a less severe environment such as respecting wildlife habitats (Barrenetxea et al., 2008; Selavo et al., 2007) or a vessel (Ceriotti et al., 2009; Kim et al., 2008), threats and rodents are remains review to the protection of wires is much more difficult than that of sensors. All of these matters make the WSN can be seen as a choice with technological advances. Furthermore, while many research efforts have been done on WSNs, and a number of introductory or prototype systems have been deployed, the data collection in sensor networks is still in its infancy and its special call for new approaches and various from other applications solutions. For example, a model of joint work in most other applications, such as monitoring of target (Gui and Mohapatra, 2004) is that the data detection or information is treated and stored locally in some nodes and can be polled later by other nodes. The sensor data collection, however, requires that all sensor data are properly collected and transmitted to the base station, so the processing of this data requires overall knowledge and is far more complex than in other applications. Using the techniques of aggregation/data fusion, this feature blocks improve network efficiency. As a result, most of the traffic sensor data collection is reported data from each sensor to the base station. This model of 'many-to-one', if not well operated traffic, will make inefficient energy consumption across the network. Case in point, the problem of energy hole has been flagged and discussed in Olariu and Stojmenovic (2006), where the sensor nodes in the vicinity of the base station are quickly exhausted through signal relay and create a gap shaped region that lets the network disconnected from the base station. One possible solution to remedy these issues is using moving entities that move round and collect data in detection zone. However, because of the toughness of the sensing environment and to minimise disruption, such a solution is often impossible in the background of sensor data collection. In addition, contrast to other sensor networks, sensors used in the collection data are frequently in large volumes and different kind (Barrenetxea et al., 2008; Ceriotti et al., 2009), the traditional thermometer, hygrometer highly specialised in the accelerometer and pressure sensor.

\subsection{Taxonomy}

In practice, the use of WSNs for data collection can be subdivided into three main stages, namely, the stage of deployment, diffusion control messages phase and data delivery stage. The deployment phase deals with issues like how to deploy the network in the detection field. Based on the request of the application, issue can be divided into deployment of coverage and deployment of location coverage, where the former requires 
that each location in the detection zone must be covered by some sensor nodes and the second requires that the sensor nodes are be tethered to specific locations by applications. In the dissemination of control messages, messages are broadcast from the base station to all sensor nodes where the challenges are situated in how to distribute messages for all sensor nodes with little transmission costs and low latency. Flooding and gossiping (Blywis et al., 2012) are two approaches commonly used diffusion that can be easily approved WSNs. So, although their basic shapes are fecklessly known, the work is improved with better efficiency while maintaining their strength in the presence of wireless transmissions mistakes. The step of providing data to the main task responds to collect sensor data. On the basis of information indicated by step 2, the sensor data assembled in different sensor nodes and delivered to the base station, where different QoS demands of different applications derive designs on various approach main considerations QoS. It is interesting to note that Phase 2 and Phase 3 can be used alternatively so that after a round of data collection, new messages are disseminated and therefore launch a new collection cycle.

\subsection{Scalable internetworking solutions}

The WSNs research should be stretches to smart grid. WSN has been a subject of active research and found numerous applications (Wheeler, 2007). Smart grid/metering seems to be a main application for WSN, especially internet of things and machine-to-machine (M2M) communication (Ilderem, 2013; Wu et al., 2011). Current efforts in the industry include IETF 6LoWPAN (Kushalnagar et al., 2007) and ROLL (Dohler et al., 2009; Kulkarni et al., 2011; Watteyne et al., 2011). Based on usage scenarios of smart meters, the global M2M network architecture, service requirements and the capabilities of the device are still to be defined. ETSI (2010) has newly developed an emerging M2M technique committee to meet these matters. The internet-working between cellular networks and LANs has received considerable attention due to the need for transparent mobility and QoS demanding. Under the smart grid, because of the very large-scale network, the characteristics of the measurement and control traffic performed in the network are not well known. For example, it could be the case when 100,000 smart meters create data traffic counters every 10 minutes. Therefore, how to design and supply a scalable solution in a trusted network so that data can be supplied to the central control utility is a timely challenge. As the traffic traverse different networks, interoperability is the wrench. In addition, some traditional research themes may need to be revised to address the movement of the grid, such as resource allocation, routing and QoS. That is because the traffic that will be created by applications of e-typical energy is probably very different from the traditional navigation/download/streaming applications that are used today with a mixture of both real-time traffic and non-real-time traffic, is produced and distributed through different parts of a smart grid (Luan et al., 2010). The interoperability of communication protocols and dedicated smart metering exchange protocols such as smart meters DLMS/COSEM (DLMS User Association, 2010) is an open research question. DLMS/COSEM standard was improved from two concepts: object modelling application data and open systems interconnection (OSI). This sufficient to cover the largest possible applications and media communication array. Work has already begun in industry attempt to resolve the transport problem DLMS data on many networks such as GPRS and PLC. Recently, the DLMS User Association has also partnered with the ZigBee Alliance and the two communities gird on 
tunnelling DLMS/COSEM on ZigBee networks to stand complicated measurement implementations. Newly, a certain number of studies on technologies for powerful power management routing were issued in the literature. An expected transmission count (ETX) based on the method of calculation of rank has been recommended for directed acyclic graph (DAG) building and maintain in RPL (Wang et al., 2010). This method improves the dependability of unicast networks AMI. The simulation results have demonstrated that the offered mechanism generates adequate shipping rate of packets and time from start to finish. In the foreseeable future, EV are considered as a major application in the smart grid, others have stressed the communication architectures for V2G. Requirements of communication and data flow between the EV and the grid were discussed, and session initiation protocol (SIP) (Li et al., 2012) is deemed as an appropriate solution for setting communications. V2G networks have also been examined in Kabish et al. (2010), concentrating on the substructure of the message and the trading message sequence between EV and the server via IPv6-based communications network controller.

\section{Data delivery approaches}

An important step in deploying a WSN is to consider the data delivery approach, how to detect and transmit data from each sensor node to the base station. Owing to the characteristic 'many-to-one' sensor data collection, network topology is often seen as a tree topology whose root is the base station, which must be pre-defined or dynamically composed so that data packets can be conveyed along. Furthermore, the existence of wireless interference and collisions poses problems in terms of programming and data packet transmissions that must be carefully treated in order to attain an effective access to wireless support. For this purpose a cross-layer design is often involved, where the MAC, network and transport layer have been together to achieve more objectives such as energy saving and reliability (Huang et al., 2013). To collect data from sensor nodes, two elements are required: maintaining the topology and transmission planning. Maintaining a topology built connected topology which maintains connectivity in the network changes and variations in link quality. The scheduler plans the packet transmission program on the basis of information from other components in order to reduce collisions and waste of energy. Considering the QoS requirements such as throughput, latency and reliability, the various optional components can be added. However, a more difficult question is that sensor nodes operate independently, so the transmission scheduling algorithm must be designed to function in a distributed way.

\subsection{Reliability}

A previous work (Xu et al., 2004) on the WSN, a system named Wisden has adopted an approach to providing data with emphasis on reliability and operated a hybrid system for reliable data supply using both recoveries hop-by-hop and end-to-end. More specifically, each node maintains a sequence number of packets received from a source node. A difference in the sequence numbers of packets received shows the loss of packets. The sequence number of the missing packet and ID source are then stored in a list of the missing and piggy-backed when a packet is transmitted. The node that echoed the lacking packet formerly will then program a retransmission, when it catches the information piggy-backed. And to allow the retransmission of recovery hop-by-hop, each new packet 
received is cached for a short period. However, if the packet loss is heavy or network topology changes due to the dynamic changes such as link quality, the hop-by-hop recovery that can fail due to temporary overflow lacking listings or lose connections to previous forwarders recovery system end-to-end is required. In particular, if a node surprises a piggy-backed list of the disappeared and missing packets found in sharing the list of same source with these packets in its own memory, it adds these packets in its own list of missing and goes on piggy-back their information in its transmissions. By these means, the information missing packages will be back hop-by-hop to reach the sources. Sources will then resend the package and complete the circle of end-to-end recoveries.

\subsection{Latency}

Wireless communication consumes an important part of the energy budgets of the sensor nodes. MAC protocols have been offered to decrease idle listening to the radio and shoot the sensor node in sleep mode to conserve more energy. These general concepts, however, if used for the collection of sensor data without scrutiny, may introduce additional latency and increase energy costs. For example, if the next-hop neighbour still asleep, a node must wait some overtime (called sleeping latency) till the neighbour becomes active. Furthermore, to reduce sleep latency, an approach adopted is to allow a node listening to potential transmissions to temporarily increase its active period for prospective input packets. However, this can make all the nodes that hear a transmission spend more time being active and consumes more collection. To minimise sleep latency and the energy costs, Kalbande (2012) proposed DMAC to improve the collection of data from sensors. The main idea is illustrated in Figure 3. Based on the network topology, the sensor nodes along a feed path from a source node to the base station starts receiving, sending, and sleep mode in sequential order. If there are more packets to forward, more data flag is piggy-backed with each preceding packet to show the next transmission. The receiver goes into receive mode, instead of going into sleep mode to listen to the next packet. In the event that receiver has more than one transmitter, the receiver is predicts that there are packets from other senders and switches to receiving. And if nothing is heard, it returns to sleep mode. In addition, within a transmission time interval, a contention-based mechanism (CSMA) is used for several senders competition for a single receiver, and another small time after each transmission is reserved for the sender which could not send to forward a small more to send packet in order to make the receiver listens to his retransmission in place of turning into sleeping mode.

Other work called STREE was proposed in Song et al. (2009), which also aims to minimise latency and reduce energy costs. Assuming the overall synchronisation, the time slot is defined as the duration of successfully transmitting a maximum transmission unit. In a unit of time, a sensor node can sleep to save energy, or perform a single task of sending or receiving. Given each sensor node has a packet to report to the base station during each cycle, for a linear topology as shown in Figure 4, an optimal schedule to minimise the length of time for data collection round is to let the even-level links and odd-level links be active alternatively, which is called wavelike forwarding. if there is a branch of topology, as shown in Figure 5, the optimum scheduling may be performed by letting the one path $\left(u_{t+k} \rightarrow u_{o}\right)$ does wavelike forwarding first, then after the link $\left(u_{t+k} \rightarrow u_{t+1}\right)$ of the path is completed, the remaining part together with the other link $\left(u_{t+k+r} \rightarrow u_{t+k+1}\right)$ will then constitute a new route and continue to wavelike forwarding. 
Figure 3 An illustration of DMAC

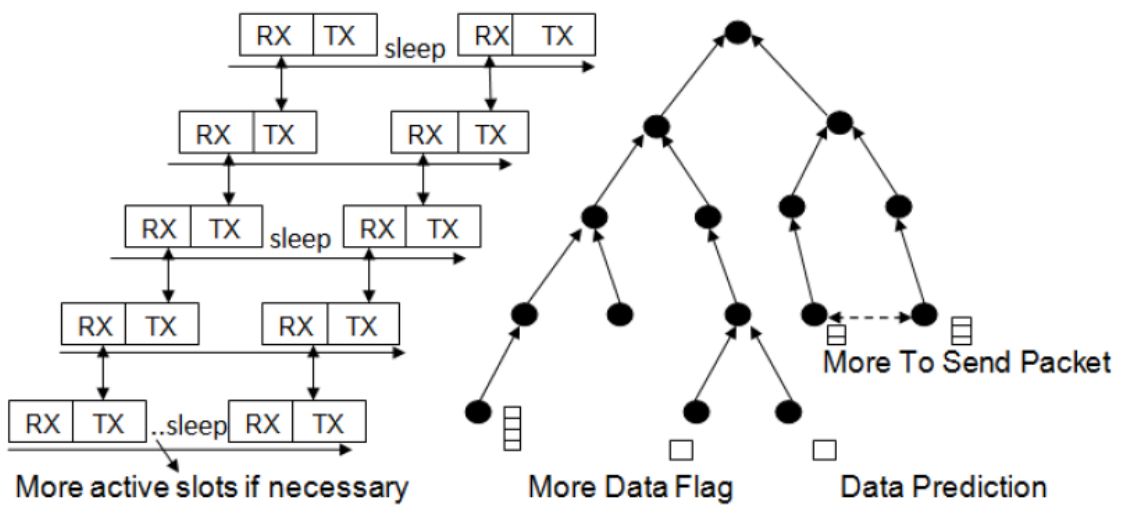

Figure 4 An illustration of time-optimal packet scheduling by STREE: linear graph

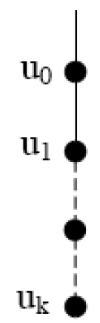

Figure 5 An illustration of time-optimal packet scheduling by STREE: simple binary tree

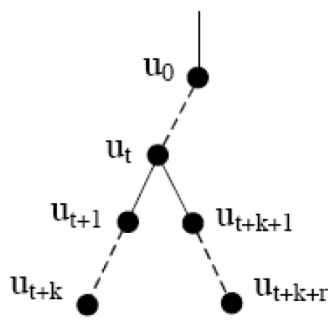

For any tree topology, a best schedule may be performed by applying recursively for each branch wave transmission. So, consider $N(u)$ the overall number of nodes in the tree anchored at $u$. The authors showed that the time for all packets from the tree rooted at $\mathrm{u}$ that can be transmitted is $2 N(u)-1$. Recently, there has been one coin of remote sensing data can be very small and multiple chunks of data can still be adapted and sent in a package to reduce the transmission overhead. This batch transmission is different from fusion aggregation technical, where multiple data are merged into a smaller size for the price of original information loss. As it is quite tedious to wait sufficient data sensor node to form a package and therefore increases the latency, Paradis and Han (2008) proposed a named TIGRA approach to batch small detection data from different sensor nodes in packets while the data browse together along the spanning tree. TIGRA uses a mechanism like rally in wave transmission introduced by Stree, but in a more general 
shape. In particular, assuming each sensor node produces a data detection and packet can contain most $m$ sensing data, TIGRA leave links whose levels are at a distance of $m$ active simultaneously, so that data transferred by remote links within distance $m$ will build in a package and then will supply to the base station. To make such a transfer mechanism for a general tree, TIGRA adopts graph colouring algorithm distributed featured to solve possible collisions and wireless interference, where the links that interfere with each other are affected by different colours so as to be active at different time slots to prevent collisions.

\subsection{Throughput}

Since the bulk of traffic in a WSN is to collect sensor data from all the sensor nodes to the base station, the more the sensor is close to the base station, more packages required to relay. To resolve this issue, funnelling-MAC illustrated in Figure 6 (Ahn et al., 2006) is offered to enhance network performance, to operate a localised control over the intensity of the region, and to make react dynamically to network conditions What addresses scalability while propose a protocol for effective planning. The main idea is to adopt a TDMA protocol in the area of traffic flow, which is served in the coverage of the issue of base station power. By monitoring traffic to the destination in every path within the region, the base station allocates time slots based on the traffic load. To maintain synchronisation, each time frame is started by a beacon from the base station, monitored by the allocation of time slots for packet transmissions.

Figure 6 An illustration of funnelling-MAC

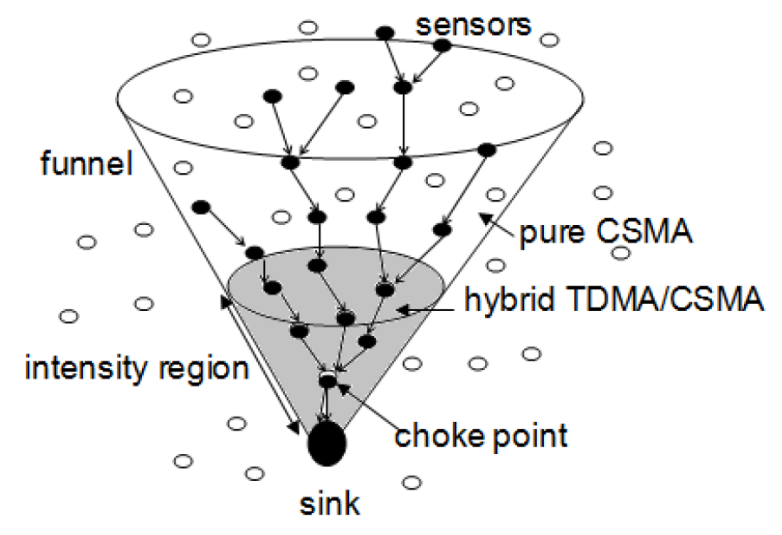

Source: Ahn et al. (2006)

The beacon transmission power is determined by the dynamic depth control algorithm. Sensors that receive a beacon become f-nodes and feel inside the intensity region. After receiving a beacon f-nodes synchronise them by initialising their own clock. Path heads operate a passive registration in which the sink aware the number of drive heads and the number of hops. If the sink realises that it can plan more nodes, it increases the transmission power of the beacon to expand the intensity zone, if the sink is aware that the number of f-nodes exceeds the maximum number of nodes that can be scheduled, it reduces the beacon transmission power. 
To assist traffic and urgency monitoring, some time slots are booked for transmission via the CSMA protocol. In addition, the base station dynamically fits the size of the region strength just a smaller size that saturates all available time slots jump. On the other hand, Sridevi et al. (2012) covered the transport layer and the solutions proposed to control congestion and equity issues. Since a tree topology routing, every node measure the mean rate $r$ to which packets can be submitted. Then, this $r$ is split by the number of sources in the descendants of the node (including itself). The result is then compared to the rating attributed by the parent and the smallest is chosen and distributed to the kids if no congestion arrives, otherwise the chosen rate is further reduced before being sent. To ensure equity, the node maintains a number of sources in the descendants of each child and uses these numbers as a weight to find the package from which the child should be sent shortly. In addition, Sridevi et al. (2012) have proposed to use non-work conservation for queues and demonstrated that although at the cost of the throughput, the non-conservation work helps reduce the risk of collision and congestion.

\subsection{Energy consumption}

As part of sensor networks, many previous works have been offered to reduce energy consumption, where data aggregation/fusion techniques are mostly used to decrease the amount of traffic issued to the base station. Due to the essential requirement for the source data, the technical data aggregation/fusion are impracticable for the sensor data collection, which requires new ways for energy-efficient. Down this direction, the authors of Burri et al. (2007) proposed, a data delivery system of ultra-low power consumption with a cross-design between MAC layer, topology control, routing and scheduling. To achieve this goal, a TDMA protocol, where a beacon is broadcasted in the early of each tower, and allocation of time slots is done for possible transmissions are adopted. During the construction phase and maintenance of the tree topology, the nodes are already included in the tags broadcast topology and assigned to slots for connection requests of the remaining nodes. The nodes receive the beacons and send connection requests to a shippers beacons and other store locally for fast recovery when the present connections fail. During the stages of the data, a parent node gives each child a separate timeslot for data communication and local synchronisation is achieved by leaving all the children listen to each beacon of the parent node. And allowing all nodes that do not listen or they are in sleeping mode, a large quantity of energy can be rescued. Moreover, to fight against collisions, a pseudo-random late jig is introduced before a beacon and is disseminated at every turn. Recently, another approach called time synchronised mesh protocol (TSMP) is available to be used in gathering sensor data (Zhen et al., 2012). TSMP calls for multi-channel communications for centralised network management. With its synchronisation mechanism enabled TSMP splits the wireless communication space along the two the time and channel dimensions as illustrated in Figure 7. A link to transmit one packet with an ACK reply to the receiver showing the reception. Every link or more link then turn one or more slots for packet transmissions. To prevent wireless interference and collisions a centralised calendar that allocates slots to links are distributed and updated as dynamic variations. Sensor nodes just rotate on their radios at slots where they are implicated in the transmission of packets according to the schedule. From wireless interference and collisions cause packet losses and retransmissions, a centralised schedule that solves the interference and losses can reduce energy consumption (Huang et al., 2013). 
Figure 7 An illustration of the matrix that divides the wireless space along both the time and channel dimensions

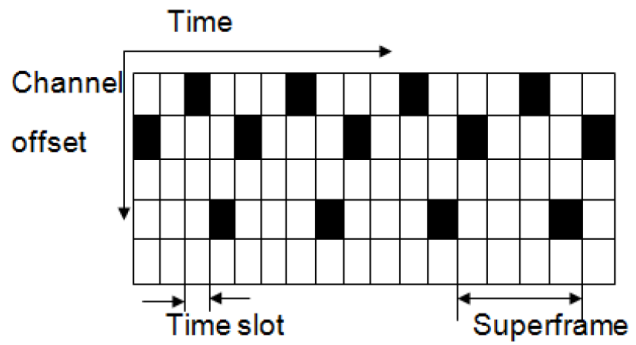

Note: Shaded locations are assigned to ties by the centralised calendar.

Source: Zhen et al. (2012)

\section{Requirements}

Monitoring/detection, communication and control are the three basic elements that converts a energy distribution system in smart grid. Monitoring/sensing has the ability to detect malfunctions or gaps from normal working ranges. Moreover, in a smart grid, a point on the electricity consumption also can be a point of generation, the detection process will be closely connected with the counting process. In the smart grid, the communications allow inputs from sensors to be transmitted to control elements which going to generate checking messages for transmission at different points. The communication infrastructure is durable enough to accept input of a user and be an integral part of the method. Similarly, the user must be able to do the level of smart grid information (Gungor et al., 2013).

\section{$6.1 Q o S$}

To achieve a convenient smart grid communication infrastructure, there is a need to have secured the QoS for communication and routing technology utilised in the smart grid.

1 Latency: communication is characterised by the fact that most interactions should be conducted in real time. The real time communications operational data in smart grid includes sensor/meter reading and power line signal control system (Fateh et al., 2013). Requirements of communication defines the design of technical solutions. For real-time detection metering, the messages should be transmitted in a very short time. For example, the maximum time allowed is about 12 to $20 \mathrm{~ms}$ depending on the type of guard, the maximum duration of a power failure fault is inside of the order of $100 \mathrm{~ms}$. Control supply system includes a monitoring control of the secondary supply process or greater levels. These systems are SCADA/EMS. The measured values should not be aged than 15 second reaching the control centre. Breaking the information must arrive by 2 seconds after the emergency (Aggarwal et al., 2010) conditions. 
2 Bandwidth: with developments of the smart grid, smart interconnected elements are added increasingly to the electricity network, the communication infrastructure should be able to carry over messages simultaneously without causing serious impacts on latency. The network bandwidth is growing faster than the demands of these smart features interlinked in the network. Hauser et al. (2008) concluded that average latency of 10 milliseconds for a message of 400 bits using a line is translated by use of only $6 \%$ of the line bandwidth. Aggarwal et al. (2010) modelled the bandwidth needs for a moderate communication system electricity distribution. In this model, a dispensing station is connected to 10,000 feeders and each supply connects to ten customers. Assuming that each electric meter produces a message every second to the distribution station, the full of 100,000 messages per second. Feeders will be generate messages to each other and to the sub-station distribution. Aggarwal et al. (2010) modelised posts in the smart grid get to servers located in the centre of traffic control by modem $\mathrm{M} / \mathrm{M} / 1$. Then, the bandwidth of the transmission line is estimated over $100 \mathrm{Mbps}$ through the $\mathrm{M} / \mathrm{M} / 1$ queue model. It may be noted that this results in a very low utilisation of bandwidth. regrettably, a higher level of use will not meet the constraint assumed latency.

\subsection{Interoperability}

The interoperability of smart grid is the ability of different systems to cooperate, utilise compatible pieces, exchange information or other equipment, and work collaboratively to complete tasks. It allows integration, effective collaboration and bidirectional communication between the interlinked elements of smart grid. The National Institute for Standards and Technology (NIST) operates as the first international coordinator for the interoperability of smart grids (Smart Grids Interoperability Standards Project). NIST has developed a framework to incorporates protocols and standards for information management to ensure interoperability of features and smart grid systems. It has expanded a three phase approach to identify the intelligent network standards.

Phase 1 focuses on the involvement of stakeholders in a participatory process to identify applicable standards and deficiencies in presently available priorities for further standardisation work, ending with the final publication of the report process on the frame after the integration of public comments.

Phase 2 will set a public-private partnership and form a board.

Phase 3 will elaborate and implement a framework for testing and certification services of how standards are applied in the smart grid features, systems and processes. As shown in Figure 8, the conceptual model NIST identifies seven areas: production, transmission, distribution, markets, operations, service, and customers, key stakeholders and applications. The model also identifies the interfaces between the domains, the actors and the applications where information should be traded and for which interoperability standards are necessary.

For many years the utilities used the optical communication port, defined by ANSI C12.18 and communication by phone modem, as defined by the ANSI C12.21 standard for measurement data. Both communication standards have been used with great success. Recent studies completed by ANSI C12.22, an interface standard for data communications network, as an update to the optical port and standard modem communication. This body of law provides the industry with an open and a full suite of 
protocols to carry the new revised standard version of the data, ANSI C12.19 (Snyder and Stuber, 2007).

Figure 8 NIST conceptual model

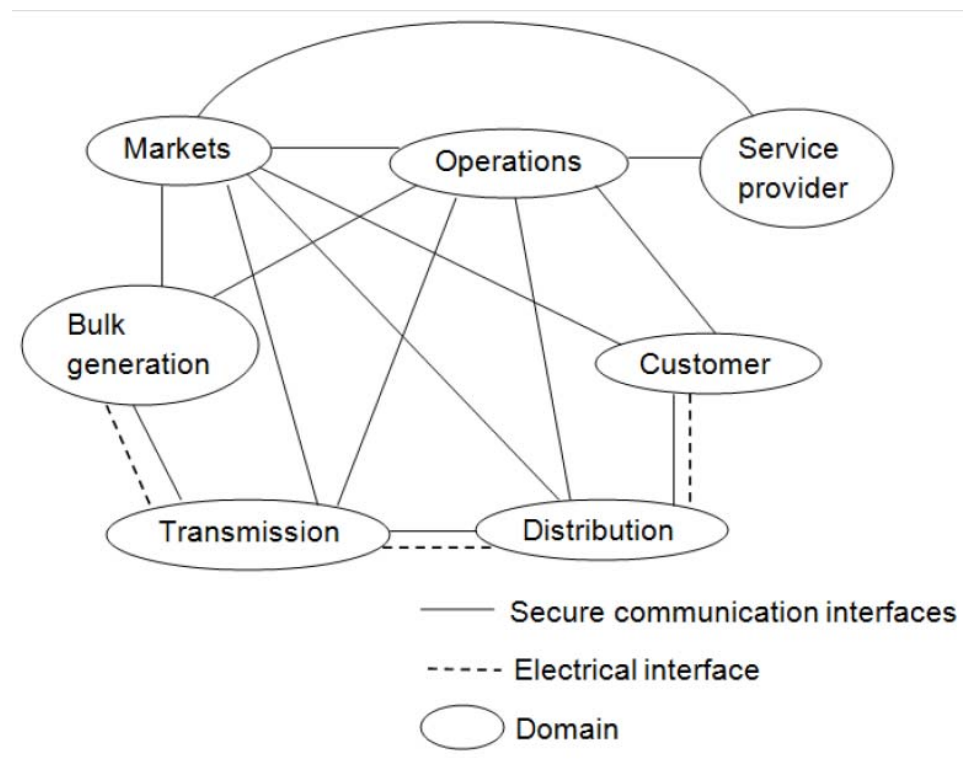

\subsection{Scalability}

A smart grid communication infrastructure purposes scalability to receive devices and services and real-time monitoring of energy meters. As reported by Lobo et al. (2008), IP network will give an efficient solution to the communication needs of the smart grid. An IP-based network as the backbone used new independent technologies and implements the service by the operator of the distributed network.

The cost of deploying and maintaining can be greatly reduced by using technologies based on IP.

\subsection{Security}

Under the Electric Power Research Institute (EPRI), an emerging demands against smart grid growing is bound to cyber security systems. As stated in the EPRI (2009) report, cyber security is a key issue because of the growing risk cyber attacks and events against this critical area as it becomes increasingly interconnected. Cyber security should deal not only intentional attacks, such disgruntled employees, the industrial spy and terrorism, but also accidentally information due to user errors, equipment failures, and natural catastrophes. Vulnerabilities permit an attacker to enter a network, access software, and change load conditions to destabilise the grid in unpredictable ways (Yan et al., 2012). There are many organisations that work on the development of smart grid safety requirements, including North American Electric Reliability Corporation-Critical Infrastructure Protection (NERC-CIP), ISA, IEEE (1402), the National Infrastructure Protection Plan (NIPP) and the NIST (Hurd et al., 2008). An important source of 
demands is the Smart Grid Interoperability Panel (SGIP) Cyber Security Working Group, previously NIST Cyber Security Coordination Task Group (CSCTG). NIST CSCTG was created to make coherence in the cyber security requirements through the fields of intelligent systems and components. A thing is consistent across different standards bodies: smart grid communications security will depend heavily on the authentication, authorisation and protection technologies. Federal Information Processing Standard (FIPS) approved Advanced Encryption Standard (AES) and Triple Data Encryption Algorithm (3DES) solutions providing security and high performance. Specific solutions needed will depend on the type communication protected resource. For example, NIST has identified that 3DES solution become probably insecure by the year 2030. Whereas the components public services are supposed to have a long life, AES would be a favourite solution for new parts. However, it is expected that features inherited as 3DES must be backed for system compatibility, so there is a risk to affect the system (Deconinck, 2010). Wire connections are insure with technologies as firewalls, virtual private networks (VPN) and IPSec. Top layer safety mechanisms such as secure shell (SSH) and security socket layer (SSL)/transport layer security (TLS) would be used. System architects and designers identify frequently the need and specify the use of security protocols such as SSH and IPsec, but unaware of the details associated with the creation of security associations. That approach is likely to lead in a system where the procedures required to ensure the management of key can rapidly become an operational nightmare. It is caused aware that when the system architects do not grow an integrated and comprehensive system key management customers can be supplied with some options key management, and frequently use hand pre-configuration symmetric keys.

\section{Challenges}

The growth of wired/wireless sensors and communication devices and the rise of board computing is an opportunity for developing connected applications, and in particular the management systems that meet the pressing challenges of the network infrastructure intelligent communications device. Challenges contain the widespread deployment of board computing, power grids heritage, smart devices and generations of communication and collaboration. In this section, we discuss the background that gives these challenges as well as emergency technical challenges that must be tackled by smart grid.

\subsection{Complexity}

A smart grid communication infrastructure is an extremely complex system. As a result, modelling, analysis and conception of a suitable communication infrastructure responds to new challenges. Models used should be able to report uncertainty as a way to simulate the behaviour emerge. The analysis should be able to solve very big scale issues. In fact, the power system is closely related and non-linear, and does not benefit from the parsimony that generally characterises this problem. The control system and in particular of the communication infrastructure must be configured to handle uncertainty and inconsistencies. Finally, performance metrics must be set to the new nature of power system. Challenges model the complexity of a communication infrastructure smart grid are summarised in the following. 
1 The need to sustain multiphysical approach: Systems are so closely interlinked, so it is not possible to just simulate the electrical subsystem (Lovett et al., 2001).

2 Need to sustain multidisciplinary approach: Several users have to work in the same scenario, each emphasising on various aspects (control, energy flows, communications) (Dougal and Monti, 2007).

3 Need to support a level of dynamic and reconfigurable pattern: This is probably the most difficult. Although different users interact with the simulation scheme they must concentrate on various details of the system. The upcoming generation simulation system should support this process automatically (Ponci et al., 2009).

4 Need to supply a graphic visualisation of high-level to sustain the system: As engineers want to concentrate on details of graphics, system scan needs various types of visualisation can synthesise a 'system-picture' (Dougal and Monti, 2004).

5 Need to supply support for the doubt spread: Uncertainty is found in the fuel system for various reasons and from different sources. And therefore, it must be taken into account in the conception and operation, it will be depend on static and dynamic rating (Rios-Zalapa et al., 2010).

\subsection{Efficiency}

The implementation of future smart grid need to meet the efficiency challenges by exploiting the technologies of communication and modern information in order to realise a communication infrastructure that supplies grid large contact with supervisory and control (Halder, 2013). The communication infrastructure must be able to provide two-way communications almost instantaneous among all devices ranging from individual loads control centres, including all the important material in electricity distribution and transmission. This implies a very large number of transactions for analysis and automation. It requires a communications infrastructure able to provide high performance quickly and intelligently provide coordinated responses with a comprehensive analysis of higher level in order to prevent the rapid development of adverse events.

1 Best ranging: The PMU technology (Amin, 2005) can provide faster, stamped, greater accuracy and scan time to allow off network to know the situation.

2 Quick controls: On the basis of the power electronics, smart grid allows fast and automated workflow management actions, voltage and power of electricity, transmission and distribution control.

3 Stronger controls: Dynamic and adaptive control parameters of protection and communication for monitoring and control of large areas.

4 Integrated intelligent device communications: To activate adaptive and intelligent communications for the device level fault diagnosis and identification of bad data, operations and limitations must be ordered by network managers or control centres, intelligent remedial action schema (RAS)/system protection systems (SPS), the self-restoration of the equipment and local self-control measures (Wen et al., 2010). 
5 Included and safe communications: Ubiquitous and highly distributed communications based on open standards to configure a flexible network monitoring and automated two-way communications between all operators and customers.

6 Improved numeracy: Secure communication system support reliable analysis and autonomous intelligent agents in time through a coordinated hierarchy (Mcgranaghan et al., 2008).

7 Internet technology: Internet protocols easier data exchange, process monitoring and computer security implements a distributed architecture with open interfaces. the hardware and software plug-and-play service-oriented architecture are based on norms and communication technologies as message-oriented middleware and web services to allow transparent inclusion of the IT infrastructure lowest equipment level intelligent electronic devices (IEDs) at all higher levels of application (Kezunovic et al., 2010).

\subsection{Reliability}

A coherent framework for the integration of technology facilitates the convergence of reliability standards and protocols and the implementation of analysis capability. we examine the impact of the communication infrastructure to the reliability of an intelligent network.

1 Renewable resources: renewable resources are generally challenges the reliability of the smart grid because of the following factors: the variability and low capacity, weak correlation with the support profile in particular in the case of the wind resource, forecast errors relatively high especially for long places, congestion in the transmission due to the large facilities and distribution level because of spread resources, operational performance problems such as voltage regulation. To face the variability of excess demand, renewable energies increasing resources in the long term, infrastructure for effective communication system for the exchange of information between demand response, storage systems and the use of plug-in electric vehicles (PEV)/plug-in hybrid electric vehicles (PHEV) will complete remedies (Mets et al., 2010).

2 Demand response: demand response enables consumer to reduce the response to emergency situations and conditions of high prices charged the intelligent network. These conditions are more common in congested operations, as shown in Figure 9. Non-emergency demand response in the range of $5 \%$ to $15 \%$ of the peak load of the system can bring substantial advantages by reducing the need for additional resources and lower electricity prices in real time (Rahimi and Ipakchi, 2011). Demand response does not significantly alter the total energy consumption because much of the energy saved during the compression is consumed at a more opportune time.

3 Charging management: the rejection of the load as urgent resource to protect the smart grid of the disturbance is including and is used to operate the system or by operator command or through sub-frequency and/or under-voltage relay. In a smart grid systems, load rejection can be strengthened to act smarter and based on client involvement. Prices based on demand/response management/load as a system of 
resources to balance the demand and supply has not been extensively yet adopted. In a smart grid, the real-time information on prices allow a wider willing consumers. Demand response can be carried out through either automatic or manual response to price signals, or through a competitive bidding process on the basis of direct communication between consumers and market operators/system or through intermediaries such as aggregators or local public services.

4 Storage systems: Majority existing storage resources are hydroelectric and pumped storage. Though, the growth potential of these resources is far smaller than the need for storage necessary to counter the variability of net increasing demand presented by new wind and solar resources. Different storage technologies are emerging to bridge the gap. Storing the battery seems to be the most promising because of technology enhancements and economies of scale. Storage resources have a tendency to the profile of the net demand and, as such, should improve reliability. In addition, storage devices can meet timescales seconds. Therefore, they can become catalysts for value control in an intelligent network. Storage resources of different sizes can be dispensed in the network from the end user to major stations and central power posts. This function can help alleviate congestion at both the transmission and dispensing levels.

Figure 9 Communications for demand response

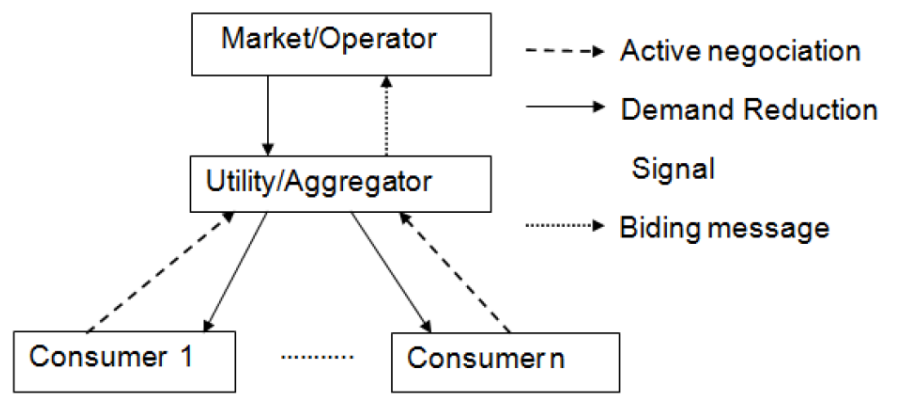

\subsection{Security}

Based on the development of infrastructure communication systems, and concern for cyber security, many new questions have been raised as part of a smart grid.

1 Information security areas: Since the SCADA/EMS systems have become more embedded, it becomes more hard to process the structure of the system in terms of parts or subsystems. Physically performing of different functions is less obvious from the point of view of the user. Instead, it becomes natural to consider a SCADA/EMS system in terms of domains. A domain is a particular area in which specific activities are underway and they can be grouped. Security domains are introduced into Figure 10 (Ericsson, 2010). When communicating through the power utilities of different organisations and companies, using communication networks, the areas of security should be recognised. For example, a company of electric utility could set a security domain, the policies and procedures of its activities. If the like definitions, procedures, policies, etc.. have been developed other utilities of 
electricity, it might be easier to discuss and establish common rules for the exchange of information or the use of common resources for a communication infrastructure. But today there are an absence of common definitions, including long-term security. In addition, there is no common drive system safety policies or procedures, although groups such as IEC (IEC, 2009), ISA (ISA 99) and NIST (Stouffer et al., 2011), are working on policies and generic procedures.

2 Public coordination on SCADA safety: Expertise collected and shared experiences, including international knowledge, have the objective of securing SCADA part of the information infrastructure. As a natural step, SCADA security guidance has been developed in Swedish Civil Contingencies Agency (2007). Also, guidelines and administrative recommendations are developed which are available for free download, which support the equity security of SCADA systems in different areas operations: electricity, water and transport.

3 Uncoupling between running SCADA/EMS and admin IT: When the current systems SCADA/EMS are retrofitted or replaced, information and computer security issues must be taken into behalf. If a SCADA/EMS system is restored, the operational part of SCADA/EMS system must be free of the administration party, so that the operation is protected against digital threats that are available on the internet. If a SCADA/EMS system is replaced, then it is a good opportunity to revisit the structure of the system and incorporate security on all levels of SCADA/EMS. One way to state this is safer to uncouple the SCADA/EMS operational and administrative computer system. In addition, an alternative may determine the configuration of the firewall between the operation and administrative documents.

4 Threatens: Is that the SCADA/EMS systems are linked and incorporated with external systems, creating new opportunities and threats in cyber security (Yan et al., 2012). Some of these new issues have been highlighted in Ericsson (2009, 2010).

5 Vulnerable: As shown in Brodsky and McConnell (2009), using a WSN in AMI in a smart grid is so vulnerable being attacked by a smart opponent, even with a ordinary frying microwave. The equipment needed for commit such attack is inexpensive (about $70 \$$ ).

6 Data protection: The data protection of limits customer and smart measurement networks is important for the possible acceptance by the public. Research in this domain is ongoing and users of smart meters will need to be assured that their data is safe. Efthymiou and Kalogridis (2010) outline a method of anonymous data safe electrical measurement frequent sent by a smart meter. Although these frequent measurement data may be required under a commercial or distribution of electric energy, these data cannot be attributed to a specific smart meter or consumer. 
Figure 10 Information security domains

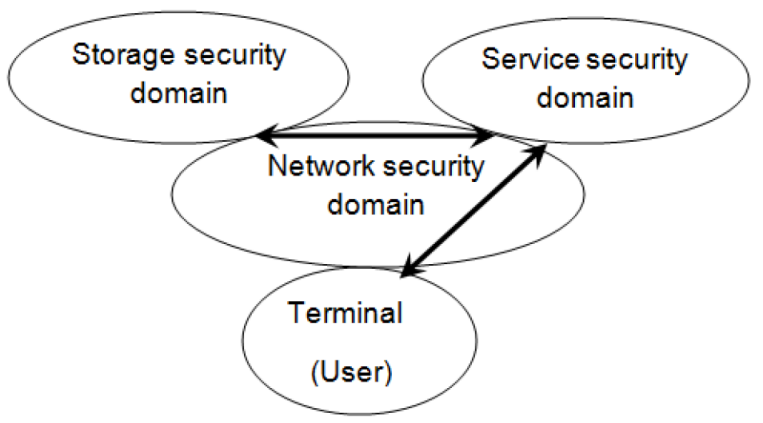

\section{Conclusions}

In this article, we presented the context of communication infrastructure networks intelligent. First, we showed that an intelligent network built on detection technologies, communication, and control technologies offer a promising future for utilities and consumers. Secondly, we talked about wireless sensor networks that have been applied to many applications since its release. Indeed, the collection of sensor data is one of the most important applications among them. In a WSN for sensor data collection, the sensor data are collected continuously all or part of sensor nodes and transmitted through wireless communications to a central base station for further treatment. This makes it different from other applications of WSN as traditional sensor data collection using wired networks. We have presented a study on recent advances in data collection in WSN. Specifically, we first identified the characteristics of the sensor data collection in WSN. Then we discussed issues on the use of sensor networks to collect data. Finally, we talked about the efficiency, reliability and safety of interconnected devices and systems that are essential to enable the communication infrastructure of smart grids. interoperability must be achieved while avoiding being isolated in non-competitive technical solutions and the need to replace existing large electrical communication systems. Tracking behind the technical standards must be balanced with the creation of an environment that encourages innovation so that the entire communication infrastructure can continue to evolve.

\section{References}

Aggarwal, A., Kunta, S. and Verma, P.K. (2010) 'A proposed communications infrastructure for the smart grid', International IEEE Conference on Innovative Smart Grid Technologies (ISGT), Gaithersburg, Maryland, pp.1-5.

Ahn, G.S., Miluzzo, E., Campbell, A.T., Hong, S.G. and Cuomo, F. (2006) 'Funneling-MAC: a localized, sink-oriented MAC for boosting fidelity in sensor networks', Proceedings of 4th ACM International Conference on Embedded Networked Sensor Systems, New York, USA, pp.293-306.

Albany, Y. (2005) The Effects of Integrating Wind Power on Transmission System Planning, Reliability and Operations, Report on Phase 2: System Performance Evaluation, The New York State Energy Research, and Development Authority.

Amin, M. (2005) 'Energy infrastructure defense systems', Proceedings of IEEE, Vol. 93, No. 5, pp.861-875. 
Amin, M. and Wollenberg, B.F. (2005) 'Toward a smart grid: power delivery for the 21st century', IEEE Journal of Power and Energy Magazine, Vol. 3, No. 5, pp.34-41.

Barrenetxea, G., Ingelrest, F., Schaefer, G. and Vetterl, M. (2008) 'SensorScope: out-of-the-box environmental monitoring', Proceedings of 8th IEEE International Conference on Information Processing in Sensor Networks, Saint Louis, Missouri, pp.332-343.

Blywis, B., Reinecke, P., Unes, M. and Wolter, K. (2012) 'Gossip routing, percolation, and restart in wireless multi-hop networks', IEEE Conference on Wireless Communications and Networking Conference (WCNC), Shanghai, China, pp.3019-3023.

Bressan, N., Bazzaco, L., Bui, N., Casari, P., Vangelista, L. and Zorzi, M. (2010) 'The deployment of a smart monitoring system using wireless sensors and actuators networks', Proceedings of 1st IEEE International Conference on Smart Grid Communications, Gaithersburg, Maryland, USA, pp.49-54.

Brodsky, J. and McConnell, A. (2009) 'Jamming and interference induced denial-of-service attacks on IEEE 802.15.4-based wireless networks', Proceedings of the SCADA Security Scientific Symposium, Washington, Surburban, pp.21-27.

Burri, N., Rickenbach, P.V. and Wattenhofer, R. (2007) 'Dozer: ultra-low power data gathering in sensor networks', Proceedings of 6th International ACM/IEEE Conference on Information Processing in Sensor Networks, Cambridge, USA, pp.450-459.

Ceriotti, M., Mottola, L., Picco, G.P., Murphy, A.L., Guna, S., Corra, M., Pozzi, M., Zonta, D. and Zanon, P. (2009) 'Monitoring heritage buildings with wireless sensor networks: the Torre Aquila deployment', Proceedings of 8th ACM/IEEE International Conference on Information Processing in Sensor Networks (IPSN), San Francisco, USA, pp.277-288.

Deconinck, G. (2010) 'Metering, intelligent enough for smart grids?', in Lukszo, Z. et al. (Eds.); Securing Electricity Supply in the Cyber Age, pp.143-157, Springer, Netherlands.

DLMS User Association (2010) [online] http://www.dlms.com (accessed 10 August 2013).

Dohler, M., Watteyne, T., Winter, T. and Barthel, D. (2009) Routing Requirements for Urban Low-power and Lossy Networks, Network Working Group, RFC 5548.

Dougal, R. and Monti, A. (2004) 'The virtual test bed concept for virtual prototyping of complex systems', Proceeding of 4th International Conference on Advanced Engineering Design, Glasgow, Scotland, Orgit Ltd, pp.1-15.

Dougal, R.A. and Monti, A. (2007) 'The virtual test bed as a tool for rapid system engineering', Proceeding of 1st IEEE Annual Conference on Systems, Honolulu, Hawaii, pp.1-6.

Efthymiou, C. and Kalogridis, G. (2010) 'Smart grid privacy via anonymization of smart metering data', Proceedings of First IEEE International Conference on Smart Grid Communications, Gaithersburg, Maryland, pp.238-243.

Electric Power Research Institute (EPRI) (2009) Report to NIST on Smart Grid Interoperability Standards Roadmap, Technical Report, Electric Power Research Institute, California [online] http://www.nist.gov/smartgrid/upload/InterimSmartGridRoadmapNISTRestructure.pdf (accessed 11 July 2013).

Ericsson, G.N. (2009) 'Treatment of information security for electric power utilities (EPUs)', IEEE Transactions on Power Delivery, Vol. 24, No. 3, pp.1174-1181.

Ericsson, G.N. (2010) 'Cyber security and power system communication essential parts of a smart grid infrastructure', IEEE Transactions on Power Delivery, Vol. 25, No. 3, pp.1501-1507.

European Telecommunications Standards Institute (ETSI) (2010) ETSI TR 102691:2010 Machineto-Machine communications (M2M) - Smart Metering Use Cases, European Union.

Fan, Z., Kulkarni, S., Gormus, S., Efthymiou, C., Kalogridis, G., Sooriyabandara, M., Zhu, Z., Lambotharan, S. and Chin, W.H. (2013) 'Smart grid communications: overview of research challenges, solutions, and standardization activities', IEEE Journal of Communications Surveys and Tutorials, Vol. 15, No. 1, pp.21-38.

Fang, X., Misra, S., Xue, G. and Yang, D. (2012) 'Smart grid - the new and improved power grid: a survey', IEEE Journal of Communications Surveys and Tutorials, Vol. 14, No. 4, pp.944-980. 
Fateh, B., Govindarasu, M. and Ajjarapu, V. (2013) 'Wireless network design for transmission line monitoring in smart grid', IEEE Journal of Smart Grid, Vol. 4, No. 2, pp.1076-1086.

Federal Energy Regulatory Commission (2010) Demand Response and Advanced Metering, Staff Report, Department of Energy, New York [online] http://www.ferc.gov/legal/staffreports/2010-dr-report.pdf (accessed 29 August 2013).

Ferreira, H., Lampe, L., Newbury, J. and Swart, T. (2010a) Power Line Communications, 1st ed., Vol. 6, No. 8, pp.3114-3123, Wiley, New York.

Ferreira, H., Lampe, L., Newbury, J. and Swart, T. (2010b) Power Line Communications: Theory and Applications for Narrowband and Broadband Communications over Power Lines, pp.1-6, John Wiley and Sons, New York.

Galli, S., Scaglione, A. and Wang, Z. (2010) 'Power line communications and the smart grid', Proceedings of First IEEE International Conference on Smart Grid Communications (SmartGridComm), Gaithersbug, Maryland, pp.303-308.

Gu, Y., Hwang, J., He, T. and Du, D.H.C. (2007) 'Sense: a unified asymmetric sensing coverage architecture for wireless sensor networks', Proceedings of 27th IEEE International Conference on Distributed Computing Systems, Toronto, Ontario, Canada, p.8.

Gui, C. and Mohapatra, P. (2005) 'Virtual patrol: a new power conservation design for surveillance using sensor networks', Proceedings of 4th International Symposium on Information Processing in Sensor Networks (IPSN), UCLA, Los Angeles, California, USA, pp.246-253.

Gungor, V.C. and Lambert, F.C. (2006) 'A survey on communication networks for electric system automation', Elsevier Journal of Computer Networks, Vol. 58, No. 2, pp.877-897.

Gungor, V.C., Lu, B. and Hancke, G.P. (2009) 'Industrial wireless sensor networks: challenges, design principles, and technical approaches', IEEE Journal of Transactions on Industrial Electronics, Vol. 56, No. 10, pp.4258-4265.

Gungor, V.C., Lu, B. and Hancke, G.P. (2010) 'Opportunities and challenges of wireless sensor networks in smart grid', IEEE Journal of Industrial Electronics, Vol. 57, No. 10, pp.3557-3564.

Gungor, V.C., Sahin, D., Kocak, T., Ergut, S., Buccella, C., Cecati, C. and Hancke, G.P. (2011) 'Smart grid technologies: communication technologies and standards', IEEE Transactions on Industrial Informatics, Vol. 7, No. 4, pp.529-539.

Gungor, V.C., Sahin, D., Kocak, T., Ergut, S., Buccella, C., Cecati, C. and Hancke, G.P. (2013) 'A survey on smart grid potential applications and communication requirements', IEEE Journal of Industrial Informatics, Vol. 9, No. 1, pp.28-42.

Halder, T. (2013) 'A case file on a smart grid', IEEE International Symposium on Industrial Electronics (ISIE), Taipei, Taiwan, pp.1-6.

Hart, D.G. (2008) 'Using AMI to realize the smart grid', Proceedings of the 21st Century IEEE Conference on Power and Energy Society General Meeting - Conversion and Delivery of Electrical Energy, Pittsburgh, Pennsylvania, USA, pp.1-2.

Hartung, C., Han, R., Seielstad, C. and Holbrook, S. (2006) 'FireWxNet: a multi-tiered portable wireless system for monitoring weather conditions in wildland fire environments', Proceedings of 4th ACM International Conference on Mobile Systems, Applications and Services, New York, USA, pp.28-41.

Hauser, C.H., Bakken, D.E., Dionysiou, I., Gjermundrod, K.H., Irava, V.S., Helkey, J. and Bose, A. (2008) 'Security, trust, and QoS in next generation control and communication for large power systems', International Journal of Critical Infrastructures, Vol. 4, Nos. 1/2, pp.3-16.

Huang, P., Xiao, L., Soltani, S., Mutka, M.W. and Xi, N. (2013) 'The evolution of MAC protocols in wireless sensor networks: a survey', IEEE Journal of Communications Surveys and Tutorials, Vol. 15, No. 2, pp.101-120.

Hurd, S., Smith, R. and Leischner, G. (2008) 'Tutorial: security in electric utility control systems', Proceeding of 61st IEEE Annual Conference on Protective Relay Engineers, Texas AM University College Station, USA, pp.304-309. 
Ilderem, V. (2013) 'Gossip routing, percolation, and restart in wireless multi-hop networks', IEEE International Symposium on VLSI Design, Automation, and Test (VLSI-DAT), Hsinchu, Taiwan, p.1.

Infield, D. and Li, F. (2008) 'Integrating micro-generation into distribution systems: a review of recent research', Proceedings of 21st Century IEEE Conference on Power and Energy Society General Meeting - Conversion and Delivery of Electrical Energy, Pittsburgh, Pennsylvania, USA, pp.1-4.

International Electrotechnical Commission (IEC) (2009) IEC 62351:2009: Power Systems Management and Associated Information Exchange Data and Communications Security Parts 1-8, Information Security for Power System Control Operations.

ISA 99 [online] http://www.isa.org/MSTemplate.cfm (accessed 5 September 2013).

Jing, L., Yang, X., Shuhui, L., Wei, L. and Philip, C.L. (2012) 'Cyber security and privacy issues in smart grids', IEEE Journal of Communications Surveys and Tutorials, Vol. 14, No. 4, pp.981-997.

Kabisch, S., Schmitt, A., Winter, M. and Heuer, J. (2010) 'Interconnections and communications of electric vehicles and smart grids', Proceedings of First IEEE International Conference on Smart Grid Communications (SmartGridComm), Gaithersburg, Maryland, pp.161-166.

Kalbande, A. (2012) 'An adaptive energy-efficient MAC protocol Guardian for wireless sensor networks', Proceedings of First IEEE international conference on Emerging Technology Trends in Electronics, Communication and Networking (ET2ECN), Surat, Gujarat, India, pp.1-5.

Kezunovic, M., Xu, F., Cuka, B. and Myrda, P. (2010) 'Intelligent processing of IED data for protection engineers in the smart grid', Proceedings of 15th IEEE Mediterranean Electrotechnical Conference on MELECON, Valletta, Malta, pp.437-442.

Kim, Y., Schmid, T., Charbiwala, Z.M., Friedman, J. and Srivastava, M.B. (2008) 'NAWMS: nonintrusive autonomous water monitoring system', Proceedings of 6th ACM Conference on Embedded Network Sensor Systems, New York, USA, pp.309-322.

Kulkarni, P., Gormus, S., Zhong, F. and Motz, B. (2011) 'A self-organising mesh networking solution based on enhanced RPL for smart metering communications', IEEE International Symposium on World of Wireless, Mobile and Multimedia Networks (WoWMoM), Lucca, Tuscany, pp.1-6.

Kushalnagar, N., Montenegro, G. and Schumache, C. (2007) IPv6 Over Low-Power Wireless Personal Area Networks (6LoWPANs): Overview, Assumptions, Problem Statement, and Goals, Network Working Group, RFC 4919.

Leon, R., Vittal, V. and Manimaran, G. (2007) 'Application of sensor network for secure electric energy infrastructure’, IEEE Journal of Power Delivery, Vol. 22, No. 2, pp.1021-1028.

Li, W., Xiong, Q. and Xu, J. (2012) 'A peer-to-peer solution for SIP servers cooperation in wireless mesh networks', Proceedings of 2nd IEEE International Conference on Consumer Electronics, Communications and Networks (CECNet), Three Gorges, Yichang, China, pp.1561-1564.

Lo, C. and Ansari, N. (2012) 'The progressive smart grid system from both power and communications aspects', IEEE Journal of Communications Surveys and Tutorials, Vol. 14, No. 3, pp.799-821.

Lobo, F., Cabello, A., Lopez, A., Mora, D. and Mora, R. (2008) 'Distribution network as communication system', IEEE Seminar on Smart Grids for Distribution (IET-CIRED), Frankfurt, Germany, pp.117-122.

Lovett, T., Monti, A., Santi, E. and Dougal, R.A. (2001) 'A multi-language environment for interactive simulation and development of controls for power electronics', Proceeding of 32nd IEEE Annual Conference on Power Electronics Specialists, Vancouver, ColombieBritannique, pp.1725-1729. 
$\mathrm{Lu}$, B. and Gungor, V.C. (2009) 'Online and remote motor energy monitoring and fault diagnostics using wireless sensor networks', IEEE Journal of Transactions on Industrial Electronics, Vol. 56, No. 11, pp.4651-4659.

Lu, B., Habetler, T.G., Harley, R.G. and Gutierrez, J.A. (2005) 'Applying wireless sensor networks in industrial plant energy management systems - part I: a closed-loop scheme', Proceedings of 4th IEEE Conference on Sensors, Irvine, California, USA, pp.145-150.

Lu, B., Habetler, T.G., Harley, R.G., Gutierrez, J.A. and Durocher, D.B. (2007) 'Energy evaluation goes wireless', IEEE Journal of Industry Applications Magazine, Vol. 13, No. 2, pp.17-23.

Luan, W., Sharp, D. and Lancashire, S. (2010) 'Smart grid communication network capacity planning for power utilities', IEEE PES Transmission and Distribution Conference and Exposition, New Orleans, USA, pp.1-4.

Mcgranaghan, M., Von Dollen, D., Myrda, P. and Gunther, E. (2008) 'Utility experience with developing a smart grid roadmap', Proceedings of 21st Century IEEE Conference on Power and Energy Society General Meeting-Conversion and Delivery of Electrical Energy, Pittsburgh, Pennsylvania, USA, pp.1-5.

Mets, K., Verschueren, T., Haerick, W., Develder, C. and De Turck, F. (2010) 'Optimizing smart energy control strategies for plug-in hybrid electric vehicle charging', IEEE/IFIP Network Operations and Management Symposium Workshops (NOMS Wksps), Osaka, Japan, pp.293-299.

Olariu, S. and Stojmenovic, I. (2006) 'Design guidelines for maximizing lifetime and avoiding energy holes in sensor networks with uniform distribution and uniform reporting', Proceedings of 27th IEEE International Conference on Computer Communications (INFOCOM), Barcelona, Spain, pp.1-12.

Paradis, L. and Han, Q. (2008) 'TIGRA: timely sensor data collection using distributed graph coloring', Proceedings of 6th IEEE International Conference on Pervasive Computing and Communications (PerCom), Hong Kong, China, pp.264-268.

Pavlidou, N., Vinck, A.H., Yazdani, J. and Honary, B. (2003) 'Power line communications: state of the art and future trends', IEEE Journal of Communications Magazine, Vol. 41, No. 4, pp.34-40.

Ponci, F., Monti, A. and Santi, E. (2009) 'Discrete-time multi-resolution modeling of switching power converters using wavelets', ACM Journal of Simulation, Vol. 85, No. 2, pp.69-88.

Rahimi, F. and Ipakchi, A. (2012) 'Demand response as a market resource under the smart grid paradigm', IEEE Transactions on Smart Grid, Vol. 1, No. 1, pp.82-88.

Rea, S., Aslam, M.S. and Pesch, D. (2013) 'Serviceware - a service based management approach for WSN cloud infrastructures', IEEE International Conference on Pervasive Computing and Communications Workshops (PERCOM Workshops), San Diego, California, pp.133-138.

Rieken, D.W. and Walker, M. (2011) 'Ultra low frequency power line communications using a resonator circuit', IEEE Journal Transactions on Smart Grid, Vol. 2, No. 1, pp.41-50.

Rios-Zalapa, R., Wang, X., Wan, J. and Cheung, K. (2010) 'Robust dispatch to manage uncertainty in real time electricity markets', IEEE Conference on Innovative Smart Grid Technologies (ISGT), Gaithersburg, Maryland, pp.1-5.

Sahu, N. and Dehalwar, V. (2012) 'Intelligent machine to machine communication in home area network for smart grid', Proceeding of Third International Conference on Computing Communication and Networking Technologies (ICCCNT), SNS College of Engineering Coimbatore, Tamilnadu, India, pp.1-6.

Samuelsson, O., Repo, S., Jessler, R., Aho, J., Karenlampi, M. and Malmquist, A. (2010) 'Active distribution network - demonstration project ADINE', IEEE Conference on Innovative Smart Grid Technologies(ISGT Europe), Lindholmen Science Park, Gothenburg, Sweden, pp.1-8.

Sauter, R. and Watson, J. (2007) 'Strategies for the deployment of microgeneration: implications for social acceptance', Elsevier Journal of Energy Policy, Vol. 35, No. 5, pp.2770-2779. 
Schelen, O., Brannstrom, R. and Ahlund, C. (2012) 'A sensor-data acquisition grid architecture', Proceedings of 10th IEEE International Conference on Networking, Sensing and Control (ICNSC), Evry, France, pp.361-366.

Schwartz, M. (2009) 'History of communications carrier-wave telephony over power lines: early history', IEEE Journal of Communications Magazine, Vol. 47, No. 1, pp.14-18.

Selavo, L., Wood, A., Cao, Q., Sookoor, T., Liu, H., Srinivasan, A., Wu, Y., Kang, W., Stankovic, J., Young, D. and Porter, J. (2007) 'Luster: wireless sensor network for environmental research', Proceedings of 5th ACM International Conference on Embedded Networked Sensor Systems, New York, USA, pp.103-116.

Serizawa, Y., Ohba, E. and Kurono, M. (2010) 'Present and future ICT infrastructures for a smarter grid in Japan', IEEE Conference on Innovative Smart Grid Technologies, ISGT, Gaithersburg, Maryland, pp.1-5.

Smart Grids Interoperability Standards Project [online] http://www.nist.gov/smartgrid (accessed 31 August 2013).

Snyder, A.F. and Stuber, M.T.G. (2007) 'The ANSI C12 protocol suite-updated and now with network capabilities', International IEEE conference on Power Systems Conference: Advanced Metering, Protection, Control, Communication, and Distributed Resources, Clemson, South Carolina, pp.117-122.

Song, W.Z., Huang, R., Xu, M., Ma, A., Shirazi, B. and LaHusen, R. (2009) 'Air-dropped sensor network for real-time high-fidelity volcano monitoring', Proceedings of 7th ACM International Conference on Mobile Systems, Applications, and Services, New York, USA, pp.305-318.

Sridevi, S., Usha, M. and Lithurin, G.P.A. (2012) 'Priority based congestion control for heterogeneous traffic in multipath wireless sensor networks', International IEEE Conference on Computer Communication and Informatics (ICCCI), Sri Shakthi Institute of Engineering and Technology, Coimbatore, India, pp.1-5.

Stouffer, K., Falco, J. and Scarfone, K. (2011) Guide to Industrial Control Systems (ICS) Security, Reports on Computer Systems Technology, Special Publication 800-82, Information Technology Laboratory, National Institute of Standards and Technology, Gaithersburg [online] http://csrc.nist.gov/publications/nistpubs/800-82/SP800-82-final.pdf (accessed 10 September 2013).

Swedish Civil Contingencies Agency (2007) Guide to Increased Security in Process Control Systems for Critical Societal Functions [online] https://www.msb.se/ (accessed 10 September 2010).

Taylor, G.A., Irving, M.R., Hobson, P.R., Huang, C., Kyberd, P. and Taylor, R.J. (2006) 'Distributed monitoring and control of future power systems via grid computing', IEEE Power Engineering Society General Meeting, Montreal, Quebec, Canada, pp.55-59.

Tenti, P., Costabeber, A. and Mattavelli, P. (2010) 'Improving power quality and distribution efficiency in micro-grids by cooperative control of switching power interfaces', International Power Electronics Conference (IPEC), Sapporo, Japan, pp.472-479.

US Department of Energy [online] http://www.oe.energy.gov (accessed 12 June 2013).

Van Loon, T., Vu Van, T., Woyte, A., Truyens, F., Bletterie, B., Reekers, J., Blazic, B. and Engelen, R. (2010) 'Increasing photovoltaics grid penetration in urban areas through active distribution systems: first large scale demonstration', Proceedings of Third International Conference on Infrastructure Systems and Services: Next Generation Infrastructure Systems for Eco-Cities (INFRA 2010), Shenzhen Library of Science and Technology, Shenzhen, China, pp.1-4.

Wang, D., Tao, Z., Zhang, J. and Abouzeid, A. (2010) 'RPL based routing for advanced metering infrastructure in smart grid', IEEE International Conference on Communications Workshops (ICC), Cape Town, South Africa, pp.1-6.

Watteyne, T., Molinaro, A., Richichi, M.G. and Dohler, M. (2011) 'From MANET to IETF ROLL standardization: a paradigm shift in WSN routing protocols', IEEE Journal of Communications Surveys and Tutorials, Vol. 13, No. 4, pp.688-707. 
Wen, J., Arons, P. and Liu, W.H.E. (2010) 'The role of remedial action schemes in renewable generation integrations', IEEE Conference on Innovative Smart Grid Technologies (ISGT), Gaithersburg, Maryland, pp.1-6.

WernerAllen, G., Lorincz, K., Johnson, J., Lees, J. and Welsh, M. (2006) 'Fidelity and yield in a volcano monitoring sensor network', Proceedings of 7th Symposium on Operating Systems Design and Implementation, Berkeley, CA, USA, pp.381-396.

Wheeler, A. (2007) 'Commercial applications of wireless sensor networks using ZigBee', IEEE Journal of Communications Magazine, Vol. 45, No. 4, pp.70-77.

Wiser, R. and Bolinger, M. (2008) Annual Report on US Wind Power Installation, Cost and Performance Trends: 2007, US Department of Energy, Energy Efficiency and Renewable Energy, USA.

Wu, G., Talwar, S., Johnsson, K., Himayat, N. and Johnson, K.D. (2011) 'M2M: from mobile to embedded internet', IEEE Journal of Communications Magazine, Vol. 49, No. 4, pp.36-43.

Xu, N., Rangwala, S., Chintalapudi, K., Ganesan, D., Broad, A., Govindan, R. and Estrin, D. (2004) 'A wireless sensor network for structural monitoring', Proceedings of 2nd ACM International Conference on Embedded Networked Sensor Systems, New York, USA, pp.13-24.

Yan, Y., Qian, Y., Sharif, H. and Tipper, D. (2012) 'A survey on cyber security for smart grid communications', International Journal of Communications Surveys and Tutorials, Vol. 14, No. 4, pp.998-1010.

Yu, R., Zhang, Y., Gjessing, S., Yuen, C., Xie, S. and Guizani, M. (2011) 'Cognitive radio based hierarchical communications infrastructure for smart grid', IEEE Journal of Network, Vol. 25, No. 5, pp.6-14.

Zhai, M.Y. (2011) 'Transmission characteristics of low-voltage distribution networks in China under the smart grids environment', IEEE Journal of Transactions on Power Delivery, Vol. 26, No. 1, pp.173-180.

Zhang, Y., Li, X., Zhang, S. and Zhen, Y. (2012) 'Wireless sensor network in smart grid: applications and issue', IEEE Conference on Information and Communication Technologies (WICT), Trivandrum, India, pp.1204-1208.

Zhen, Y., Hui-chuan, D. and Lin, Q. (2012) 'Prolonging time synchronized mesh protocol network's lifetime by adjusting transmission range and routing graph', International IEEE Symposium on Information Technology in Medicine and Education (ITME), Future University Hokodate, Hokkaido, Japan, pp.910-913. 